\title{
Modelling bubble clusters in compressible liquids
}

\author{
D. Fuster $\dagger$ and T. Colonius \\ Division of Engineering and Applied Science, California Institute of Technology, Pasadena, \\ CA 91125, USA
}

(Received 3 March 2011; revised 6 July 2011; accepted 7 September 2011; first published online 21 October 2011)

We present a new model for bubbly cavitating flows. Based on volume-averaged equations, a subgrid model is added to account for a bubble, or multiple bubbles, within each computational cell. The model converges to the solution of ensembleaveraged bubbly flow equations for weak oscillations and monodisperse systems. In the other extreme, it also converges to the theoretical solution for a single oscillating bubble, and captures the bubble radius evolution and the pressure disturbance induced in the liquid. A substantial saving of computational time is achieved compared to ensemble-averaged models for polydisperse mixtures.

Key words: bubble dynamics, cavitation, multiphase flow

\section{Introduction}

\subsection{Motivation}

The modelling and simulation of bubbly flows has been a challenging problem in fluid dynamics since Lord Rayleigh started his first investigations on the behaviour of bubbles exposed to external pressure changes (Rayleigh 1917). The complexity of the phenomena involved in gas/liquid mixtures impedes rigorous theoretical analysis in common situations encountered in industry and nature, such as ship propellers, underwater explosions and biomedical applications. Only under a large number of assumptions can one find analytical or semi-analytical solutions that provide, in the most favourable case, conditions to validate numerical results (Foldy 1945; Commander \& Prosperetti 1989). The numerical solution of the equations is not simple either. The high computational cost related to the direct numerical simulation (DNS) of the Navier-Stokes equations (Seo, Lele \& Tryggvason 2010) impedes accurate simulations of bubble collapse involving many bubbles.

In processes where cavitation is important, initial nuclei grow rapidly due to evapouration when the pressure is near or below the vapour pressure. Bubbles that initially have radii on the order of microns reach a maximum radius from hundreds of microns to few millimetres. These bubbles often occur in clusters whose length scale is much larger that the bubble radius (typical sizes of clusters range from tens of centimetres to metres). The bubbles generated in the regions of low pressure

$\dagger$ Email address for correspondence: fuster@dalembert.upmc.fr

$\$$ Present address: CNRS (UMR 7190), Université Pierre et Marie Curie, Institut Jean le Rond d'Alembert, France 
then collapse when the pressure is increased. The pressure recovery is either as a consequence of the bubble advection from the region of low pressure or because of temporal variations of the pressure at the bubble location induced by incoming waves.

Owing to the difficulties of performing DNS (Delale \& Tryggvason 2008), several simplified models for bubbly flows have been proposed in the literature. Thanks to these models, various mechanisms playing a role in the behaviour of the mixture have been identified. Evapouration and condensation processes affect vapour content inside the bubbles during expansion as well as the bubble radius evolution (Storey \& Szeri 2000; Puente \& Bonetto 2005). Compressibility effects in the liquid play an important role in the pressure wave propagation. The pressures waves are not only generated by external disturbances, but also from the cluster itself (Reisman, Wang \& Brennen 1998; Wang \& Brennen 1999). When bubbles collapse, the pressures reached inside the bubbles are large enough to induce shock waves in the liquid.

In addition, there are various phenomena inside the bubbles that can have a significant impact on the bubble behaviour: chemical reactions (Hauke, Fuster \& Dopazo 2007), transient effects related to the mass transfer (Fuster, Hauke \& Dopazo 2010), and internal pressure waves (Xu et al. 2003) are a few examples of the complex phenomena that can influence the maximum pressures and temperatures reached during the collapse. Another important mechanism that significantly influences these peak pressures and temperatures is non-spherical bubble collapse. Simulations of non-spherical bubbles (Oguz \& Prosperetti 1990; Chahine 1993; Bergmann et al. 2006; Delale \& Tryggvason 2008; Johnsen \& Colonius 2009; Wang \& Blake 2010) typically consider the dynamics of one or two bubbles. Thus, although they are useful to gain insight into the mechanisms of interactions of a few bubbles (Bremond et al. 2006a), the simplifications applied impede an understanding of all the mechanisms related to the propagation of pressure waves through bubble clusters with current computational resources.

Various experimental studies have been aimed at improving the understanding of the response of bubble clusters. Mettin \& Lauterborn (2003) have shown that the presence of a few bubbles is enough to significantly modify the pressure waves. Bremond et al. (2006b) show that the behaviour of each individual bubble in the cluster is influenced by the behaviour of the surrounding bubbles. They also show that modified versions of the Rayleigh-Plesset (RP) equations already capture the main mechanisms of bubble-bubble interactions in what they call the 'weak interaction regime'. These studies exemplify the two-way coupling problem. On one hand, the presence of bubbles changes the structure of the incoming pressure wave, even when the wavelength is much larger than the bubble size. The changes depend on, among other factors, the void fraction that, in turn, depends on how the bubble responds. On the other hand, the bubble response is influenced by the large scale variations in imposed pressure and by the disturbances induced by the surrounding bubbles. This two-way coupling problem stresses the importance of the modelling of the effects on both small and large scales. The main goal of this work is to present a model that efficiently captures the two-way coupling effect and which may be used in future investigations of the response of bubble clusters.

\subsection{The current approach}

A model is developed to resolve individual bubbles that are larger than the grid spacing and to model the effect of a cluster of bubbles that is smaller than the grid spacing. 
The volume-averaged Navier-Stokes equations (see for example Commander \& Prosperetti 1989) are solved in a three-dimensional domain that is discretized in space. The void fraction, defined as the volume of gas per unit volume of mixture, is obtained locally for each cell by projecting the volume of the bubbles on to the grid. Bubbles are considered to remain spherical; therefore, the model is strictly valid for systems with bubbles with a small Weber number, where surface tension effects are important enough to keep bubbles spherical. In addition, the solution of the current model can be considered as an approximation in conditions where nonspherical effects, though present, are of secondary importance. The validity of this assumption has been widely discussed in the literature experimentally, as in Lauterborn \& Bolle (1975), computationally, see for example Best \& Kucera (1992) and Blake et al. (1999), and theoretically (a review of classical theories can be found in Brennen 1995 and references therein).

Bubbles are tracked as Lagrangian particles. In the examples contained here, we focus on bubble displacements that are small relative to the characteristic size of the bubble cluster. In any event, the model could, in principle, include relative motion with modelled forces on the bubbles (as for example those presented in Tomar et al. 2010). Similar models have been successfully applied to predict the motion of bubble clusters in acoustic cavitation (Hinsch 1976; Parlitz et al. 1999). The heat and mass transfer across the interface is modelled using the model proposed by Preston, Colonius \& Brennen (2007).

A subgrid model, based on a potential flow hypothesis, is used to resolve the detailed dynamics in the vicinity of bubbles. The disturbances induced by the bubbles on the averaged flow field are captured by decomposing the potential generated inside each cell into the potential induced by the background flow and the potential induced by each individual bubble present in the cell. The potential induced by the background flow is constant inside a cell, whereas the potential generated by each bubble decays with the distance from the bubble centre. Therefore, for each cell we can define a distance beyond which the potential induced by the bubbles contained in the cell is negligible. This position defines an effective pressure at infinity for each cell. By applying the same potential decomposition, a new extension of the RP equation is derived for bubble clusters in compressible liquids. The new equation tends to the limiting case of the Keller-Miksis equation (Keller \& Miksis 1980) for a single bubble, and to the equation used by previous authors for bubble clusters in incompressible liquids (Bremond et al. 2006b).

As we show, the new model is able to capture the effect of phenomena like pressure wave propagation, relative bubble motion, and viscous effects. The model is also able to reproduce the behaviour of the individual bubbles present in the liquid, capturing the individual pressure waves scattered and generated during bubble dynamics. This fact extends the applicability of the current model to a wider variety of problems where DNS is presently unfeasible. In the next sections, the model is derived in detail.

\section{Previous models}

The simulation of bubble clusters dates back to Foldy (1945) who presented a general theory for scattering systems in the linear regime. In the 1960s, Iordanskii (1960), Kogarko (1964) and van Wijngaarden (1964, 1968) independently developed similar models to capture the effect of nonlinear oscillations. In his review, Prosperetti (1997) summarizes the main contributions of van Wijngaarden in the context of bubble 
cluster modelling. Defining averaged values for the density and velocity of the mixture, these models are based on the assumption that 'the average pressure $p$ is the pressure at infinity of single-bubble theory' which, as stated by Prosperetti, 'contains the insight that makes the whole machinery work'.

Typical models for disperse gas/liquid mixtures are based on the presence of bubble nuclei in the liquid. These nuclei are assumed to be spherical and remain much smaller than the length scale upon which the averaged properties of the flow vary. Nowadays, two widely used models for disperse bubbly flows are based on the volume-averaged equations and on the ensemble-averaged equations.

\subsection{Volume-averaged equations}

Models based on the volume-averaged equations are designed to find an approximate solution of a given two-phase problem where one of the phases can be considered as disperse. By defining $\bar{\rho}$ and $\overline{\boldsymbol{u}}$ as the average local mixture density and centre-of-mass velocity, the equations of continuity and momentum for a mixture can be written, in the absence of external sources of momentum, in their usual form (Commander \& Prosperetti 1989):

$$
\begin{gathered}
\frac{\mathrm{D} \bar{\rho}}{\mathrm{D} t}=-\bar{\rho} \nabla \cdot \overline{\boldsymbol{u}}, \\
\bar{\rho} \frac{\mathrm{D} \overline{\boldsymbol{u}}}{\mathrm{D} t}=-\nabla p+\nabla \cdot \boldsymbol{\tau},
\end{gathered}
$$

where $\tau$ can be seen as the averaged mixture viscous stress and $p$ as an averaged pressure of the mixture.

Caflisch et al. (1985) rigorously prove that the velocity of the bubbles can be neglected at the order of the approximations introduced in the model, so that $\overline{\boldsymbol{u}}$ can be approached by the velocity of the liquid $\boldsymbol{u}_{l}$.

The average local mixture density is defined by using the void fraction, $\beta$, which in this case expresses the amount of gas per unit volume. According to this definition the averaged density for liquid/gas mixtures can be approximated for mixtures where the gas is considered as the dilute phase as

$$
\bar{\rho}=\rho_{l}(1-\beta)+\rho_{g} \beta \approx \rho_{l}(1-\beta),
$$

where subscripts $l$ and $g$ denote liquid and gas phase respectively. Commander \& Prosperetti (1989) show how this formulation is able to recover the previous heuristic Wijngaarden-Papanicolaou model, given a relation between the pressure and the density, $\mathrm{d} P=c^{2} \mathrm{~d} \rho$, where $c$ is the speed of sound in the liquid.

Finally, the value of the void fraction is defined as

$$
\beta(\boldsymbol{x}, t)=\frac{4}{3} \pi \int_{0}^{\infty} R^{3}\left(R_{0} ; \boldsymbol{x}, t\right) f\left(R_{0} ; \boldsymbol{x}\right) \mathrm{d} R_{0},
$$

where $R$ is the bubble radius, $R_{0}$ represents the equilibrium bubble radius and $f\left(R_{0} ; \boldsymbol{x}\right)$ is the number density of bubbles with equilibrium radius between $R_{0}$ and $R_{0}+\mathrm{d} R_{0}$ in the neighbourhood of point $\boldsymbol{x}$.

\subsection{Ensemble-averaged equations}

As a generalization of models based on volume-averaged equations, Zhang \& Prosperetti (1994a) propose a systematic method to derive averaged equations for disperse two-phase flows. This methodology can be applied to systems where one of the phases is considered as disperse (Zhang \& Prosperetti 1994b, 1997; Marchioro, 
Tanksley \& Prosperetti 2000). For the particular case of bubbly flows the final equations obtained from this analysis are known as ensemble-averaged equations (Zhang \& Prosperetti 1994b). Similar approaches have been used by Akhatov et al. (1997) and Akhatov, Parlitz \& Lauterborn (1996) and extended models have been proposed by Tanguay (2003) and Ando, Colonius \& Brennen (2009, 2011). These models are designed to capture the changes in the structure and the speed of the waves that propagate throughout the bubble cluster. Theoretical models based on linear acoustic theory (Commander \& Prosperetti 1989) are already able to capture some of these effects, showing an excellent agreement with the numerical computations performed by Ando et al. $(2009,2011)$. However, these models still resort to a large number of assumptions. The most relevant ones are that mutual interactions among the bubbles are neglected except through their effect on the mixture-averaged flow, length scales in the (averaged) mixture are large compared to the mean bubble spacing, bubbles advect with the ambient liquid velocity, and density and velocity fluctuations in the liquid phase are uncorrelated (Ando et al. 2009).

These equations are designed to find the average response of a more general two-phase problem. In this case, the solution of the system of equations provides information about the averaged behaviour of the mixture for an infinite number of particular problems, where bubbles obeying a known probability radius distribution, $f_{0}\left(R_{0}\right)$, are randomly distributed over a given region of space. When looking at the final formulation of the equations, the main difference with the volume-averaged equations stems from the definition of the void fraction. In this case, $\beta$ is interpreted as a probabilistic distribution which indicates the probability of having a given value of the void fraction at a given location and it is defined as

$$
\beta=\frac{4}{3} \pi n \overline{R^{3}},
$$

where $n$ is the number of bubbles per volume of mixture and $\overline{R^{3}}$ can be obtained from the probabilistic function of the bubble radius distribution

$$
\overline{R^{3}}=\int_{0}^{\infty} R_{0}^{3} f_{0}\left(R_{0}\right) \mathrm{d} R_{0} .
$$

Unlike (2.4), this equation does not explicitly account for the spatial distribution of bubbles. Instead, it considers the probability of finding a bubble of given radius $R_{0}$ over an infinite number of repetitions.

Finally, we need to define an equation to relate the averaged pressure and density. For linear waves in bubbly flows, we can use the acoustic relation (Commander \& Prosperetti 1989)

$$
\mathrm{d} \bar{\rho}=\bar{c}^{-2} \mathrm{~d} p .
$$

\subsection{Detailed models for the bubble contents}

The radial oscillation of the bubbles is typically obtained using variations of the RP equation. Most of the equations proposed in the literature to obtain the temporal evolution of the bubble radius are based on a potential flow hypothesis. Equations for the bubble dynamics require a boundary condition for the pressure far from the bubble that must ultimately be determined in terms of the local pressure in the volume- or ensemble-averaged equations. Under the incompressible liquid assumption, these equations can be generalized to account for the presence of the surrounding bubbles. In this case, some correction terms (named bubble-bubble interaction terms) 
are introduced in the formulation if the pressure far from the bubble cloud is known (Bremond et al. 2006b; Ilinskii, Hamilton \& Zabolotskaya 2007).

These models correctly reproduce the degree of expansion of a small number of bubbles in the weak interaction regime (Bremond et al. 2006b), but they miss some important effects in clouds with a large number of bubbles, namely, wave propagation in the mixture, damping effects induced by liquid compressibility during the bubble collapse, and vorticity in the mixture. Shell methods (Seo et al. 2010) appear as a remedy for some of these problems. By discretizing in space the averaged equations, the equation describing the radial motion of the bubble is written as a function of the pressure at a finite distance (shell radius), where the pressure, in turn, is associated with the mixture. The main drawback of shell methods is that they cannot be easily extended to compressible liquids, where an analytical expression for the pressure at a given distance from the bubble cannot be obtained. In addition, the computation of the shell for every bubble contained in the domain can be expensive in the general case where the bubbles move. Finally, another family of RP-like equations includes liquid compressibility effects in the formulation (Gilmore 1952; Keller \& Miksis 1980). However, these equations still assume that the pressure far from the bubble is known and they do not account for the presence of the surrounding bubbles.

The pressure inside the bubble is determined by the model used for the gas phase. The main difference between models comes from how heat and mass transfer across the interface is included. The simplest models consider uniform properties inside the bubble and assume that the gas behaves adiabatically or isothermally. Different models have been proposed in the literature to account for the effect of heat transfer (Devin 1959; Chapman \& Plesset 1970; Prosperetti, Crum \& Commander 1988) although an exhaustive validation of these models when intense evapouration takes place is missing.

To include mass transfer effects in these models is not a simple task. The diffusion of water vapour inside the bubble usually controls the evaporation flux when the pressure of the system falls below the liquid vapour pressure. For an accurate prediction of the bubble growth, the mass flux must be taken into account. Different models have been presented in the context of single-bubble dynamics including evapouration/condensation across the interface (Yasui 1997; Storey \& Szeri 2000; Xu et al. 2003; Hauke et al. 2007). However, these models solve for the internal concentration gradients inside the bubble and result in expensive computations even for the case of a single spherical, oscillating bubble. As an alternative, Preston et al. (2007) have proposed a reduced-order model to predict the mass flux across the interface. We use this model here, but note that in principle it is easy to replace with higher-fidelity models should they become available.

The motion of the bubbles is modelled by either solving a transport equation for the bubble number density (in ensemble-averaged models) or using Lagrangian methods, the latter considering each bubble as a physical point whose displacement can be obtained from Newton's law. The different forces acting on the bubble due to relative motion with the liquid may be modelled. Magnaudet \& Eames (2000) present a complete review of available models. For the particular case of bubbles in liquids, the works by Parlitz et al. (1999) and Climent \& Magnaudet (2006) are two relevant examples of experimental studies of the influence of different forces on the bubble motion. The drag force, lift force, Bjerknes forces, and inertial force are some examples of typical forces usually considered to predict the bubble motion. The relevance of each of them strongly depends on the problem of interest and particular 
studies are required to determine which forces can be neglected. In principle, any of these models can be utilized in the model we present in the next section.

\section{New model}

\subsection{Liquid equations}

Using the definition of the averaged density (2.3), we can rewrite (2.1) and (2.2) as

$$
\begin{gathered}
\frac{\partial \rho_{l}}{\partial t}+\nabla \cdot\left(\rho_{l} \boldsymbol{u}\right)=\frac{\rho_{l}}{1-\beta}\left[\frac{\partial \beta}{\partial t}+\boldsymbol{u} \cdot \nabla \beta\right], \\
\frac{\partial \rho_{l} \boldsymbol{u}}{\partial t}+\nabla \cdot\left(\rho_{l} \boldsymbol{u} \boldsymbol{u}\right)+\frac{\nabla p}{1-\beta}=\frac{\nabla \cdot \boldsymbol{\tau}}{1-\beta}+\frac{\boldsymbol{u} \rho_{l}}{1-\beta}\left[\frac{\partial \beta}{\partial t}+\boldsymbol{u} \cdot \nabla \beta\right],
\end{gathered}
$$

where, following Caflisch et al. (1985), we neglect the effect of the gas phase on the velocities so that $\overline{\boldsymbol{u}}=\boldsymbol{u}_{l} \equiv \boldsymbol{u}$. This formulation is convenient for numerical purposes because the effects induced by the changes in the void fraction are grouped on the right-hand sides of the equations, which allows splitting techniques to be utilized (Toro 1997; LeVeque 2002).

The main difference among all the models based on the volume-averaged equations is the interpretation and definition of the values of the void fraction, $\beta$, and the pressure, $p$. The definition of these values ultimately determines the range of applicability of the model. Therefore, it is important to carefully choose the model and method used to define these quantities. In the following sections, these models are described.

\subsection{Gas-phase equations: void fraction}

The Lagrangian treatment of the bubbles as particles preserves the mass of gas. This mass defines a void fraction field over a given region of space. The main departure of the present model from previous ones is the procedure developed to obtain $\beta$ from (2.4) given the location of the bubbles. Unlike the models based on the ensembleaveraged equations, the function $f\left(R_{0} ; \boldsymbol{x}\right)$ depends not only on $R_{0}$, but also on the coordinate $\boldsymbol{x}$. Thus, the calculation of $f\left(R_{0} ; \boldsymbol{x}\right)$ given a known distribution of bubbles in the system turns out to be too involved. As a workaround it is possible to approximate the void fraction field by invoking the notion of a kernel approximation. This concept has been successfully applied to different systems of partial differential equations. Monaghan (1982) provides a theoretical background for these methods in the context of smooth particle hydrodynamics (SPH). Following these developments, the void fraction is approximated as

$$
\beta \approx \sum_{i} \frac{4}{3} \pi R_{i}^{3} \Upsilon(d, h),
$$

where $d=\left|\boldsymbol{x}-\boldsymbol{x}_{i}\right|$ is the distance from the bubble location $\boldsymbol{x}_{i}, h$ is a measure of the size of the support (a regularization parameter), and $\Upsilon$ is the kernel function. The selection of the kernel function is restricted by five conditions enumerated in Monaghan (1982) and Belytschko et al. (1996) but in principle both the kernel function and the size of the support are two degrees of freedom introduced in the model. A variety of possible kernels can be found in Peskin (2003). The use of this approximation can be interpreted as an artifact to project the volume of the bubbles whose location and volume is known, over an arbitrary region of space $\Omega$ whose characteristic length scale is controlled by the parameter $h$. The size of the support allows modelling 
bubbles with a radius larger than the mesh size, $\Delta x$, by imposing $h>R(t)>\Delta x$. When the bubble radius is smaller than the cell size, it is desirable to define the local void fraction on the smallest volume possible in order to better approach the real concentration field. As will be shown later, a discrete delta function for the kernel function provides the most accurate results in the limit of a single bubble. The exact expression for the kernel can be found in Peskin (2003).

Using this approximation, the temporal derivative of $\beta$ is

$$
\frac{\partial \beta}{\partial t}=\sum_{i}\left[\frac{\partial V_{i}}{\partial t} \Upsilon(d)+V_{i} \frac{\partial \Upsilon(d)}{\partial t}\right],
$$

where $V_{i}=(4 / 3) \pi R_{i}^{3}$. As we only allow spherical oscillations, the temporal derivative is directly given by

$$
\frac{\partial V_{i}}{\partial t}=4 \pi R_{i}^{2} \dot{R}_{i}
$$

Finally, applying the chain rule, the temporal derivative of the symmetric kernel can be expressed as

$$
\frac{\partial \Upsilon(d)}{\partial t}=-\boldsymbol{u}_{p} \cdot \nabla \Upsilon(d)
$$

where $\boldsymbol{u}_{p}$ denotes the bubble velocity represented as a Lagrangian particle.

\subsection{The bubble dynamic model}

In this work, a new RP-like equation is proposed to predict the bubble dynamics of a bubble cluster in a compressible liquid. This equation tries to overcome the limitations of some previous RP-like equations. The method is designed to obtain the bubble radius evolution of bubbles whose radius approaches the order of the cell size only using the information in the bubble surroundings. In this case, the pressure of the cell cannot be considered to be representative of the pressure far from the bubble and some correction terms must be introduced in the formulation in order to recover the solution for a single bubble in a field where the pressure far from the bubble is known. Liquid compressibility effects and the bubble-bubble interaction effects are taken into account.

The procedure followed to derive the equation of motion is a generalization of the method developed by Keller \& Miksis (1980) for single bubbles. In this case, the model is generalized to account for the presence of surrounding bubbles and to those situations where the pressure at a finite distance is known. As proposed by Keller \& Miksis (1980), the solution of the wave equation for the potential is usually a good approximation of the solution of the continuity and momentum equations in the liquid immediately surrounding the bubbles. Taking advantage of the linear properties of this equation, the potential in the liquid is decomposed into the sum of the potential created by the background flow, $\phi_{\infty}$, and the potential induced by each of the $N_{\text {cell }}$ bubbles present in the cell: $\phi_{i}$,

$$
\phi=\phi_{\infty}+\sum_{i=1}^{N_{\text {cell }}} \phi_{i} .
$$

By properly imposing the boundary conditions at the interface of every bubble contained in the cell, it is possible to derive an equation of motion for each bubble. 
Details about the full derivation can be found in the Appendix. A simplified version of the equation is

$$
\left(R_{i}\left(1-\frac{\dot{R}_{i}}{c}\right)\right) \ddot{R}_{i}+\frac{3}{2} \dot{R}_{i}^{2}\left(1-\frac{\dot{R}_{i}}{3 c}\right)=\left(H_{i}+\frac{\partial \phi_{\infty}}{\partial t}\right)\left(1+\frac{\dot{R}_{i}}{c}\right)+\frac{R_{i} \dot{H}_{i}}{c}+I^{*},
$$

where

$$
\begin{gathered}
I^{*}=\sum_{j \neq i}^{N_{\text {cell }}}\left[\left(1+\frac{\dot{R}_{i}}{c}\right) \frac{\partial \phi_{j}\left(R_{i}\right)}{\partial t}\right]-\sum_{j \neq i}^{N_{\text {cell }}}\left[\left(1+\frac{\dot{R}_{j}\left(t^{\prime}\right)}{c}\right) \frac{\partial \phi_{j}\left(t^{\prime}-R_{j}\left(t^{\prime}\right) / c\right)}{\partial t^{\prime}} \frac{\dot{R}_{j}}{c} \frac{R_{i}}{R_{j}}\right], \\
\frac{\partial \phi_{i}\left(R_{i}\right)}{\partial t}=-\frac{1}{2} \dot{R}_{i}^{2}-H_{i}-\sum_{j \neq i}^{N_{\text {cell }}} \frac{\partial \phi_{j}\left(R_{i}\right)}{\partial t}-\frac{\partial \phi_{\infty}}{\partial t}
\end{gathered}
$$

and $R_{i}, \dot{R}_{i}, \ddot{R}_{i}$ are respectively the bubble radius, velocity and acceleration, $c$ is the speed of sound of the liquid and $H_{i}$ is the enthalpy of the liquid at the interface that can be obtained as

$$
H_{i}=\int_{p_{0}}^{p_{i}} \frac{\mathrm{d} p}{\rho},
$$

where $p_{i}$ is the pressure at the interface and $p_{0}$ is the reference pressure.

Equation (3.8) tends to the limiting case of the Keller and Miksis equation (Keller \& Miksis 1980) for a single isolated bubble in an infinite field, and it also recovers the bubble-bubble interaction term used in previous works in the incompressible limit.

To solve this equation, $\partial \phi_{\infty} / \partial t$ at the location of the bubble must be known. The method for determining it from the local pressure in computational cells is discussed in the next section.

\subsection{The background potential}

Equation (3.8) requires $\partial \phi_{\infty} / \partial t$ as a driving term for the motion of the bubble. This value is not directly given by the solution of the volume-averaged conservation equations and, therefore, an expression for the background potential as a function of the averaged values is obtained in this section. The liquid pressure inside a cell is related to its density by the state equation

$$
p_{l}=f_{p}\left(\rho_{l}\right) .
$$

On the other hand, the averaged properties are defined with respect to the volume in which the kernel function is applied, $\Omega$. Thus,

$$
\int \bar{\rho} \mathrm{d} \Omega=\sum_{i=1}^{N} \rho_{b i} \frac{4}{3} \pi R_{i}^{3}+\int_{\Omega_{l}} \rho_{l} \mathrm{~d} \Omega_{l} \approx \int_{\Omega_{l}} \rho_{l} \mathrm{~d} \Omega_{l},
$$

where $N$ is the number of bubbles contained in $\Omega$ and $\Omega_{l}$ represents the region of $\Omega$ occupied by the liquid (see figure 1).

The flow inside $\Omega$ is idealized as an irrotational flow and the Bernoulli equation is used to relate the properties of any pair of points inside it. For small density variations in the liquid (over the scale of a cell), the Bernoulli equation is

$$
\frac{\partial \phi}{\partial t}+\frac{1}{2}(\nabla \phi)^{2}+\frac{p_{l}-p_{0}}{\rho_{l, 0}}=0 .
$$




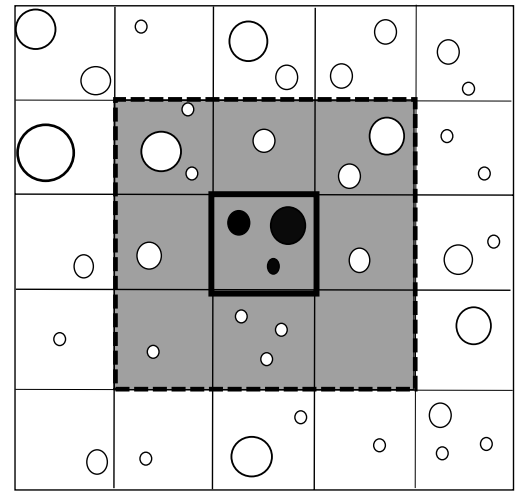

FIGURE 1. For a given cell, a representation of $\Omega$ (volume inside the dashed line) and $\Omega_{l}$ (shaded region excluding the bubbles). The bubbles contained inside the cell are plotted in black $\left(N_{\text {cell }}=3\right)$ and the total number of bubbles inside $\Omega$ is $N=15$.

It is convenient to integrate the Bernoulli equation over $\Omega_{l}$, where the background potential is approximately constant. Inside a cell, the potential is decomposed according to (3.7); therefore, we can obtain an averaged value of $\partial \phi_{\infty} / \partial t$ as

$$
\frac{\partial \phi_{\infty}}{\partial t}=-\frac{1}{V_{l}} \sum_{i=1}^{N_{\text {cell }}} \int_{\Omega_{l}} \frac{\partial \phi_{i}}{\partial t} \mathrm{~d} \Omega_{l}-\frac{1}{V_{l}} \int_{\Omega_{l}} \frac{1}{2}\left(\sum_{i=1}^{N_{\text {cell }}} \nabla \phi_{i}\right)^{2} \mathrm{~d} \Omega_{l}-\frac{p_{\text {cell }}-p_{0}}{\rho_{l, 0}},
$$

where

$$
V_{l}=\int \mathrm{d} \Omega_{l}
$$

and we have defined the value of $p_{\text {cell }}$ as

$$
p_{\text {cell }}=\frac{1}{V_{l}} \int_{\Omega_{l}} p_{l} \mathrm{~d} \Omega_{l} .
$$

On the other hand, we can write $N_{\text {cell }}$ equations evaluated at the interface of each bubble located inside the cell. In particular, for the bubble $j$

$$
\frac{\partial \phi_{\infty}}{\partial t}+\sum_{i=1}^{N_{\text {cell }}} \frac{\partial \phi_{i}\left(\boldsymbol{x}=\boldsymbol{x}_{j}\right)}{\partial t}+\frac{1}{2} \dot{R}_{j}^{2}+\frac{p_{j}-p_{0}}{\rho_{l, 0}}=0
$$

Using (3.15), $\partial \phi_{\infty} / \partial t$ can be expressed as a function of the potential $\partial \phi_{i} / \partial t$. Thus, it is possible to find a solution of the $N_{\text {cell }}$ values of $\partial \phi_{i} / \partial t$ by solving the following system of equations:

$$
\begin{aligned}
& \sum_{i=1}^{N_{\text {cell }}}\left(\frac{\partial \phi_{i}\left(\boldsymbol{x}=\boldsymbol{x}_{j}\right)}{\partial t}-\frac{1}{V_{l}} \int_{\Omega_{l}} \frac{\partial \phi_{i}}{\partial t} \mathrm{~d} \Omega_{l}\right)-\frac{1}{2} \frac{1}{V_{l}} \int_{\Omega_{l}}\left(\sum_{i=1}^{N_{\text {cell }}} \nabla \phi_{i}\right)^{2} \mathrm{~d} \Omega_{l} \\
& \quad=-\frac{1}{2} \dot{R}_{j}^{2}+\frac{p_{\text {cell }}-p_{j}}{\rho_{l, 0}} .
\end{aligned}
$$


To obtain an approximate value of the integral terms, we define the notion of a surrogate bubble that represents the global effect of all the bubbles contained inside the cell. The volume of this bubble is the sum of the volumes of the $N_{\text {cell }}$ bubbles $\left(V_{b}=\sum_{i=1}^{N_{\text {cell }}} V_{i}\right)$ so that an equivalent radius is defined as $R_{b}=\left(3 V_{b} / 4 \pi\right)^{1 / 3}$. In this case, the potential generated by the bubble in its surroundings is modelled under the assumption that the flow near the bubble is approximately incompressible; therefore,

$$
\overline{\phi_{b}}(t, r)=\frac{g(t)}{r} .
$$

At the interface of this bubble, the temporal derivative is defined as the average over the area of all the bubbles present in the cell

$$
\frac{\partial \overline{\phi_{b}}\left(t, R_{b}\right)}{\partial t}=\frac{1}{R_{b}} \frac{\partial g(t)}{\partial t}=\frac{1}{\sum_{i=1}^{N_{\text {cell }}} R_{i}^{2}} \sum_{i=1}^{N_{\text {cell }}} R_{i}^{2} \frac{\partial \phi_{i}\left(t-R_{i} / c\right)}{\partial t},
$$

whereas the radial derivative is

$$
\frac{\partial \overline{\phi_{b}}\left(t, R_{b}\right)}{\partial r}=\frac{-g(t)}{R_{b}^{2}}=\frac{\sum_{i=1}^{N_{\text {cell }}} R_{i}^{2} \dot{R}_{i}}{\sum_{i=1}^{N_{\text {cell }}} R_{i}^{2}} .
$$

Using these definitions, the integrals for the contribution of the bubbles contained in $\Omega$ are approximated as

$$
\begin{aligned}
\frac{1}{V_{l}} \int_{\Omega_{l}} \sum_{i=1}^{N_{\text {cell }}} \frac{\partial \phi_{i}(t-r / c)}{\partial t} \mathrm{~d} \Omega_{l} & \approx \frac{1}{V_{l}} \int_{R_{b}}^{R_{\Omega}} \frac{\partial \phi_{b}(t, r)}{\partial t} 4 \pi r^{2} \mathrm{~d} r \\
& =\frac{3}{2} \frac{1}{\sum_{i=1}^{N_{\text {cell }}} R_{i}^{2}} \sum_{i=1}^{N_{\text {cell }}} R_{i}^{2} \frac{\partial \phi_{i}\left(t-R_{i} / c\right)}{\partial t} \frac{R_{b}\left(R_{\Omega}^{2}-R_{b}^{2}\right)}{R_{\Omega}^{3}-R_{b}^{3}},
\end{aligned}
$$

where $R_{\Omega}=\left((3 / 4 \pi) \int \mathrm{d} \Omega\right)^{1 / 3}$ represents the equivalent radius of the volume on which the kernel is applied.

Analogously, for the integral involving the radial derivative of the potential, we write

$$
\begin{aligned}
\frac{1}{V_{l}} \int_{\Omega_{l}} \frac{1}{2}\left(\sum_{i=1}^{N_{\text {cell }}} \nabla \phi_{i}\right)^{2} \mathrm{~d} \Omega_{l} & \approx \frac{1}{V_{l}} \int_{R_{b}}^{R_{\Omega}} \frac{1}{2}\left(\frac{\partial \phi_{b}}{\partial r}\right)^{2} 4 \pi r^{2} \mathrm{~d} r \\
& =\frac{3}{2}\left(\frac{R_{b}^{3}}{R_{\Omega}^{3}-R_{b}^{3}}\right)\left(\frac{\sum_{i=1}^{N_{\text {cell }}} R_{i}^{2} \dot{R}_{i}}{\sum_{i=1}^{N_{\text {cell }}} R_{i}^{2}}\right)^{2}\left(1-\frac{R_{b}}{R_{\Omega}}\right) .
\end{aligned}
$$


Substituting (3.23)-(3.24) into (3.19), one obtains a system of $N_{\text {cell }}$ equations where the equation for bubble $j$ is

$$
\begin{aligned}
& \sum_{i=1}^{N_{\text {cell }}}\left[\frac{\partial \phi_{i}\left(t-R_{i} / c\right)}{\partial t}\left(\frac{R_{i}}{d_{i j}}-\frac{3}{2} \frac{R_{b}\left(R_{\Omega}^{2}-R_{b}^{2}\right)}{R_{\Omega}^{3}-R_{b}^{3}} \frac{R_{i}^{2}}{\sum_{\text {cell }}^{N_{k}} R_{k}^{2}}\right)\right] \\
& =\frac{3}{2}\left(\frac{R_{b}^{3}}{R_{\Omega}^{3}-R_{b}^{3}}\right)\left(\frac{\sum_{i=1}^{N_{\text {cell }}} R_{i}^{2} \dot{R}_{i}}{\sum_{i=1}^{N_{\text {cell }}} R_{i}^{2}}\right)^{2}\left(1-\frac{R_{b}}{R_{\Omega}}\right)-\frac{1}{2} \dot{R}_{j}^{2}+\frac{p_{\text {cell }}-p_{j}}{\rho_{l, 0}},
\end{aligned}
$$

where $d_{i j}$ is the distance between bubbles $i$ and $j$ when $i \neq j$, and it is 1 when $i=j$.

Note that the method is exact for the case of a single bubble located in a cell. For cells containing more than one bubble, the method to obtain the integrals can be considered as only an approximation of the real value. Despite the simplifications the method provides good results for different coarsening levels ranging from one bubble per cell, to a few bubbles per cell. For cells containing a large number of bubbles, it is shown below that the solution of the system above can be neglected in a first approximation and therefore, there is no need to apply any approximation to evaluate the integral terms in (3.19).

\subsection{The meaning of the pressure at infinity}

Once the temporal derivative of the background potential is obtained, it is possible to define a state for every cell where the potential of the bubbles has no influence. The pressure on the liquid in this region far from the bubbles can be obtained from the Bernoulli equation as

$$
p_{\infty, i}=p_{0}-\rho_{l, 0} \frac{\partial \phi_{\infty}}{\partial t}=p_{\text {cell }}+\rho_{l, 0} I^{* *},
$$

where we have defined the quantity $I^{* *}$ as

$$
I^{* *}=\frac{1}{V_{l}} \sum_{i=1}^{N_{\text {cell }}} \int_{\Omega_{l}} \frac{\partial \phi_{i}}{\partial t} \mathrm{~d} \Omega_{l}+\frac{1}{V_{l}} \int_{\Omega_{l}} \frac{1}{2}\left(\sum_{i=1}^{N_{\text {cell }}} \nabla \phi_{i}\right)^{2} \mathrm{~d} \Omega_{l} .
$$

It is interesting to note that from (3.26), when $I^{* *}$ is negligible compared to the pressure in the cell $\left(I^{* *} \ll p_{\text {cell }} / \rho_{l, 0}\right)$ then the pressure at infinity is directly given by the pressure at the cell $\left(p_{\infty} \approx p_{\text {cell }}\right)$. That is, the pressure at the cell node, $p_{\text {cell }}$, represents the pressure at infinity when the contribution of the potential induced by the bubbles is negligible in a scale of the order of the cell size. Otherwise, when $I^{* *} \sim p_{\text {cell }} / \rho_{l, 0}$, the pressure far from the bubble is not well represented by $p_{\text {cell }}$. To gain further insight, we write the driving term in (3.8) as

$$
D=\left(H_{i}+\frac{\partial \phi_{\infty}}{\partial t}\right)\left(1+\frac{\dot{R}_{i}}{c}\right)+\frac{R_{i} \dot{H}_{i}}{c}+I^{*},
$$


which, for $\dot{R}_{i} \ll c$, simplifies to

$$
D=\frac{p_{i}-p_{\infty}}{\rho_{l, 0}}+I^{*}=\frac{p_{i}-p_{\text {cell }}}{\rho_{l, 0}}+I^{*}-I^{* *} .
$$

When only one bubble resides inside a given cell $\left(I^{*}=0\right)$, the term $I^{* *}$ must be used to correctly predict the bubble dynamics. However, in situations where there are a large number of small bubbles compared to the mesh size, the potential contribution of bubble $i$ to $I^{* *}$ is negligible. In this case, $I^{*}$ represents the potential induced by the $N_{\text {cell }}-1$ surrounding bubbles at the location of bubble $i$, which on average is expected to be similar to the averaged contribution of the $N_{\text {cell }}-1$ surrounding bubbles at an arbitrary point of the cell. Thus, as $\dot{R}<c$,

$$
I^{*}=\sum_{j \neq i}^{N_{\text {cell }}} \frac{\partial \phi_{j}\left(R_{i}\right)}{\partial t} \approx \frac{1}{V_{l}} \sum_{i=1}^{N_{\text {cell }}} \int_{\Omega_{l}} \frac{\partial \phi_{i}}{\partial t} \mathrm{~d} \Omega_{l},
$$

and the term $I^{*}-I^{* *}$ becomes of the order of the volume-averaged value of the kinetic energy in the liquid

$$
I^{*}-I^{* *} \approx \frac{1}{V_{l}} \int_{\Omega_{l}} \frac{1}{2}\left(\sum_{i=1}^{N_{\text {cell }}} \nabla \phi_{i}\right)^{2} \mathrm{~d} \Omega_{l} .
$$

This equation provides a measure of the accuracy of the approximation

$$
D \approx \frac{p_{i}-p_{\text {cell }}}{\rho_{l, 0}} .
$$

In general the influence of the disturbances induced by the surrounding bubbles on the kinetic energy, represented by $(\partial \phi / \partial r)^{2}$, decays with $r_{i j}^{-4}$, whereas the term $I^{*}$, which represents the disturbances on the potential, decays with $r_{i j}$. Therefore $\left|I^{*}-I^{* *}\right|$, which is of the order of $(\partial \phi / \partial r)^{2}$ is much smaller than $I^{*}$. This result implies that in a first approximation there is no need to solve a coupled system of equations for those cells containing a large number of bubbles when the local void fraction is lower than a certain threshold. In this case, (3.32) will give a reasonable estimation not only of the pressure at the cell, but also of the dynamics of every individual bubble. For a small number of bubbles whose size is comparable to the cell size, the system of equations (3.25) must be solved if an accurate prediction of the bubble radius is desired. From a practical point of view then, we will only solve for (3.25) when the number of bubbles inside the cell is smaller than 10 . Otherwise, the driving term in (3.8) is directly given by (3.32), so the solution of the system (3.25) is not required.

\subsection{Conservation equations inside the bubble}

The reduced model proposed by Preston et al. (2007) is used to obtain the properties inside the bubble. Heat and mass transfer across the interface are modelled using the coefficients obtained from a linear analysis.

\subsection{Two-dimensional model}

The equations above define the set of equations required to solve the three-dimensional problem. However, it is also interesting to extend the model to flows that are twodimensional on average. To do this, we define an average bubble spacing in the $z$-direction, $L_{z}$. In a general case, the value of $L_{z}$ is a new degree of freedom of the 
problem. However, for problems with an isotropic distribution of bubbles, we consider that $L_{z}$ is proportional to the averaged bubble spacing which is defined as

$$
L_{z}=C\left(\frac{(4 / 3) \pi \overline{R^{3}}}{\beta}\right)^{1 / 3},
$$

where $\overline{R^{3}}$ is given by (2.6) and $C$ is a constant that for the simulations contained in this work is set to 1 .

The position of the bubbles is defined in a three-dimensional space $\left(x_{b}, y_{b}, z_{b}\right)$ with $z_{b} \in\left[0: L_{z}\right]$, but only two-dimensional displacements are permitted. Thus, the interaction terms can be obtained in a three-dimensional space, whereas the void fraction is obtained by projecting the volumes of the bubbles onto the two-dimensional plane as

$$
\beta=\sum \frac{4}{3} \pi R_{i}^{3} \frac{\gamma_{2 D}}{L_{z}}
$$

where $\gamma_{2 D}$ is a two-dimensional kernel that must be divided by the length on the spanwise direction in order to obtain the gas volume per unit volume.

\section{Summary of equations}

As a summary of the equations of the model, we solve the volume-averaged equations

$$
\begin{gathered}
\frac{\partial \rho_{l}}{\partial t}+\nabla \cdot\left(\rho_{l} \boldsymbol{u}\right)=\frac{\rho_{l}}{1-\beta}\left[\frac{\partial \beta}{\partial t}+\boldsymbol{u} \cdot \nabla \beta\right], \\
\frac{\partial \rho_{l} \boldsymbol{u}}{\partial t}+\nabla \cdot \rho_{l} \boldsymbol{u} \boldsymbol{u}+\frac{\nabla p}{1-\beta}=\frac{\nabla \cdot \boldsymbol{\tau}}{1-\beta}+\frac{\boldsymbol{u} \rho_{l}}{1-\beta}\left[\frac{\partial \beta}{\partial t}+\boldsymbol{u} \cdot \nabla \beta\right],
\end{gathered}
$$

where the pressure and the void fraction are defined as

$$
\begin{aligned}
& \beta=\sum_{i} \frac{4}{3} \pi R_{i}^{3} \Upsilon(d, h), \\
& p=p_{0}+\left(\rho_{l}-\rho_{l, 0}\right) c^{2} .
\end{aligned}
$$

Equations (4.1)-(4.4) are a closed system of equations if the location, radius, velocity and acceleration of each individual bubble present in the system are known.

The motion of the individual spherical bubbles is obtained from point forces, or in some simplified cases, by merely advecting the bubbles with the velocity of the fluid. The radial oscillation is obtained from

$$
\left(R_{i}\left(1-\frac{\dot{R}_{i}}{c}\right)\right) \ddot{R}_{i}+\frac{3}{2} \dot{R}_{i}^{2}\left(1-\frac{\dot{R}_{i}}{3 c}\right)=\left(H_{i}+\frac{\partial \phi_{\infty}}{\partial t}\right)\left(1+\frac{\dot{R}_{i}}{c}\right)+\frac{R_{i} \dot{H}_{i}}{c}+I^{*},
$$

where

$$
\begin{gathered}
I^{*}=\sum_{j \neq i}^{N_{\text {cell }}}\left[\left(1+\frac{\dot{R}_{i}}{c}\right) \frac{\partial \phi_{j}\left(R_{i}\right)}{\partial t}\right]-\sum_{j \neq i}^{N_{\text {cell }}}\left[\left(1+\frac{\dot{R}_{j}\left(t^{\prime}\right)}{c}\right) \frac{\partial \phi_{j}\left(t^{\prime}-R_{j}\left(t^{\prime}\right) / c\right)}{\partial t^{\prime}} \frac{R_{i}}{R_{j}} \frac{\dot{R}_{j}}{c}\right] \\
\frac{\partial \phi_{i}\left(R_{i}\right)}{\partial t}=-\frac{1}{2} \dot{R}_{i}^{2}-H_{i}-\sum_{j \neq i}^{N_{\text {cell }}} \frac{\partial \phi_{j}\left(R_{i}\right)}{\partial t}-\frac{\partial \phi_{\infty}}{\partial t}
\end{gathered}
$$


The equations above require the evaluation of the potential derivatives of each bubble, which can be obtained solving the following system of $N_{\text {cell }}$ equations for the bubbles contained in each cell:

$$
\begin{aligned}
& \sum_{i=1}^{N_{\text {cell }}}\left[\frac{\partial \phi_{i}\left(t-R_{i} / c\right)}{\partial t}\left(\frac{R_{i}}{d_{i j}}-\frac{3}{2} \frac{R_{b}\left(R_{\Omega}^{2}-R_{b}^{2}\right)}{R_{\Omega}^{3}-R_{b}^{3}} \frac{R_{i}^{2}}{\sum_{k=1}^{N_{\text {cell }}} R_{k}^{2}}\right)\right] \\
& =\frac{3}{2}\left(\frac{R_{b}^{3}}{R_{\Omega}^{3}-R_{b}^{3}}\right)\left(\frac{\sum_{i=1}^{N_{\text {cell }}} R_{i}^{2} \dot{R}_{i}}{\sum_{i=1}^{N_{\text {cell }}} R_{i}^{2}}\right)^{2}\left(1-\frac{R_{b}}{R_{\Omega}}\right)-\frac{1}{2} \dot{R}_{j}^{2}+\frac{p_{\text {cell }}-p_{j}}{\rho_{l, 0}},
\end{aligned}
$$

where $R_{\Omega}=\left((3 / 4 \pi) \int \mathrm{d} \Omega\right)^{1 / 3}, R_{b}=\left(\left(3 \sum_{i=1}^{N_{\text {cell }}} V_{i}\right) / 4 \pi\right)^{1 / 3}, p_{\text {cell }}$ is defined only as a function of $p$ in (3.17) and $p_{j}$ is the pressure of the $j$ th bubble obtained from the state equation of the gas and the solution of the thermal model equations.

Once the potential of every single bubble is obtained, the background potential is readily obtained as

$$
\begin{aligned}
\frac{\partial \phi_{\infty}}{\partial t}= & -\frac{3}{2} \frac{1}{\sum_{i=1}^{N_{\text {cell }}} R_{i}^{2}} \sum_{i=1}^{N_{\text {cell }}} R_{i}^{2} \frac{\partial \phi_{i}\left(t-R_{i} / c\right)}{\partial t} \frac{R_{b}\left(R_{\Omega}^{2}-R_{b}^{2}\right)}{R_{\Omega}^{3}-R_{b}^{3}} \\
& -\frac{3}{2}\left(\frac{R_{b}^{3}}{R_{\Omega}^{3}-R_{b}^{3}}\right)\left(\frac{\sum_{i=1}^{N_{\text {cell }}} R_{i}^{2} \dot{R}_{i}}{\sum_{i=1}^{N_{\text {cell }}} R_{i}^{2}}\right)^{2}\left(1-\frac{R_{b}}{R_{\Omega}}\right)-\frac{p_{\text {cell }}-p_{0}}{\rho_{l, 0}} .
\end{aligned}
$$

\section{Limiting cases}

\subsection{Single isolated bubble}

The current model solves the volume-averaged equations in a discretized space. By construction, the method is designed to provide averaged values of the pressure of the liquid at each grid cell, not the values of the liquid properties far from the bubble. The equation of motion of the bubble, and, in particular, the temporal derivative of the background potential, is derived to reproduce the pressure variations at a point far from the bubble from the local information contained in the cell. The method is derived to be exact for the limit of a single bubble, and this property is demonstrated in test problems in $\S 7$. Thus, the current method is able to recover both the pressure distributions around the bubble $\left(p_{\text {cell }}\right)$ and the bubble radius evolution of an isolated bubble where the pressure far from it is known $\left(p_{\infty}\right)$. This approximation holds even for bubbles that become of the order of the mesh size, when the disturbances induced by the bubble on a length scale of the order of the mesh size are appreciable. 


\subsection{Convergence to ensemble-averaged models}

This section discusses the range of convergence of the current model to ensembleaveraged models. In ensemble-averaged models, the void fraction is defined according to (2.5), where the value $\overline{R^{3}}$ is obtained from the probability distribution using (2.6). The pressure of the system, which is usually interpreted as $p_{\infty}$, is obtained from the state equation in the liquid

$$
p=f_{p}\left(\rho_{l}\right)=f_{p}\left(\frac{\bar{\rho}}{1-\beta}\right)=f_{p}\left(\frac{\bar{\rho}}{1-4 / 3 \pi n \overline{R^{3}}}\right) .
$$

In the current approach, the pressure at the cell node is defined as

$$
p_{\text {cell }}=f_{p}\left(\rho_{l}\right)=f_{p}\left(\frac{\bar{\rho}}{1-\sum_{i=1}^{N} \frac{4}{3} \pi R_{i}^{3} \Upsilon(d, h)}\right),
$$

whereas the rest of the equations in the two models under comparison are similar. Aside from considerations about the different nature of the average procedures, it can be concluded that both models give the same solution when

$$
(4 / 3) \pi n \overline{R^{3}}=\sum_{i=1}^{N} \frac{4}{3} \pi R_{i}^{3} \Upsilon(d, h) .
$$

We can integrate this expression over an arbitrary volume $V_{c}$ which contains $N_{c}$ bubbles and is larger than the volume over which the kernel is applied,

$$
\overline{R^{3}} n V_{c}=\sum_{i=1}^{N_{c}} R_{i}^{3},
$$

where we have applied one of the properties that must be satisfied by the kernel function,

$$
\int_{V_{c}} \Upsilon(d, h)=1 .
$$

Typically, we will take $h$ as the cell size, so that $V_{c}$ is the cell volume of a regular mesh and $N_{c}$ is the number of bubbles contained in the cell $N_{\text {cell }}$. However, we should note that $h$ is in principle a degree of freedom of the model; larger values may be useful in different situations.

Clearly both models recover the same solution if a sufficiently large control volume $V_{c}$ is considered, so that the averaged value of the quantity $\sum_{i=1}^{N_{c}} R_{i}^{3}$ is represented well by $n \overline{R^{3}}$. Both models tend to the same solution when the level of spatial accuracy demanded for the current model is low enough. This conclusion is not surprising. For high spatial resolutions, ensemble-averaged models are not expected to capture the exact pressure at a given location whereas the current model is designed to do that. For low spatial resolutions, the current model only captures the effects of direct bubble-bubble interactions by means of the interaction terms appearing in the RP-like equation (3.8). For weak oscillations and dilute systems, low-spatial-resolution simulations should converge to the same solution as the ensemble-averaged models for 
a relatively homogeneous distribution of bubbles. As the spatial resolution is increased, the comparison between the pressure provided by ensemble-averaged models and the real pressure at a given location for a given problem is expected to have some uncertainty related to the fluctuations induced by the individual bubbles that cannot be captured by the ensemble-averaged equations.

\section{Numerical method}

\subsection{General procedure}

The main purpose of the current paper is the development of a model to simulate bubbly flows. For the simulations contained in this work the model has been implemented in a base compressible flow code because it was readily available, but the model may be coupled to different types of flow solvers.

In our case, the three-dimensional averaged equations (3.1)-(3.2) are discretized in space and solved for the density and velocity fields using a skew-symmetric formulation in generalized orthogonal coordinates (Honein \& Moin 2004; Mattsson \& Nordström 2004; Franck \& Colonius 2010). The method uses high-order-accurate finite-difference methods in the streamwise and wall-normal directions, and a Fourier method for derivatives in the spanwise direction. The right-hand sides of (3.1)-(3.2) are decoupled from the rest of the averaged equations following the splitting scheme proposed by Toro (1997) (LeVeque 2002).

A fourth-order Runge-Kutta (RK) method is used to advance the left-hand side of (3.1)-(3.2), whereas an adaptive fifth/sixth-order RK method (Press et al. 1992) is used to advance the right-hand side. The splitting method has been verified to provide accurate solutions by comparison with the coupled solution of the averaged equations and the equations for the gas. The main difference here is that for the cases where implosions are especially violent, the time step for the RK scheme is adapted according to the time step of the adaptive RK in the previous step. This feature turns out to be important in order to accurately capture the pressure waves generated during the bubble implosions.

In order to obtain the temporal derivatives of the potential of each bubble the method requires the solution of a system of $N_{\text {cell }}$ equations for each cell (3.25), where $N_{\text {cell }}$ is the number of bubbles present in this cell. This system is efficiently solved by implementing the libraries for the solutions of linear systems provided by Intel Math Libraries (Schenk, Waechter \& Hagemann 2007; Schenk, Bollhöfer \& Römer 2008). Once the potentials of each cell are obtained, $\partial \phi_{\infty} / \partial t$ is obtained using (3.18), and the acceleration of the interface of each bubble contained in the system is given by (3.8).

The void fraction is obtained by applying (3.3). For efficient and fast access to the information related to the individual bubbles, a dynamic circular linked list is defined for each cell of the domain. This structure is complemented with a dynamic list of all the cells containing bubbles. Both structures must be updated every time a bubble changes from one cell to another.

\section{Verification test cases}

In the previous section we have seen that there are certain limiting cases to which the solution of the complete model should converge. In the next sections, we use these limiting cases as verification cases of the code. 


\subsection{Isolated bubble}

In this section the accuracy of the method in the limit of a single bubble is studied. This comparison is not straightforward. As previously explained, the current method is designed to capture the pressure disturbances induced by the bubble on its surroundings. Therefore, the pressure at the bubble location does not correspond, in a general case, to the pressure at infinity. We remark that the driving pressure is still defined locally, which means that only the information contained in the bubble surroundings is required to work out the bubble radius evolution. This feature saves a significant amount of computational time.

As the mesh is refined, the bubble diameter becomes of the order of the mesh size and the differences between the cell pressure and the pressure at infinity become evident. In this case, the pressure at the cell node is significantly influenced by the presence of the bubble and the pressure at infinity and the pressure at the node is different.

As a validation test the following example is considered. A single gas bubble initially in equilibrium with a given pressure $\left(p_{0}\right)$ is excited by a planar sinusoidal wave that is sent towards the bubble. In the absence of a bubble, the pressure at the bubble location can be described as

$$
\left.\begin{array}{ll}
p_{l}=p_{0}+\Delta p_{\infty} \sin (2 \pi f t) & 0<t<\frac{1}{f}, \\
p_{l}=p_{0} & t>\frac{1}{f},
\end{array}\right\}
$$

where $p_{0}=1 \mathrm{~atm}, \Delta p_{\infty}=2 \mathrm{~atm}$, and $f=150 \mathrm{kHz}$. A single bubble with an initial radius equal to $R_{0}=50 \mu \mathrm{m}$ is placed at the centre of a three-dimensional simulation domain with dimensions $2 L \times L \times L$, where $L=200 R_{0}$.

A regular Cartesian mesh is used where the mesh size, $\Delta x$, is set such that $\Delta x=4 R_{0}$. This example is especially interesting because the size of the bubble is comparable to the mesh size; therefore, the integral terms in (3.25) are no longer negligible and the consistency of the method for large void fractions, as well as in the limit of a single bubble, can be investigated.

For sufficiently weak oscillations, the theoretical solution of this problem can be approximated by solving the one-dimensional problem of a single bubble in a compressible liquid whose pressure far from the bubble evolves according to (7.1). The one-dimensional problem provides not only information regarding conditions inside the bubble, but also the pressure waves generated around it. The pressure around the bubble at a given instant $t$ and position $r$ can be obtained by the Bernoulli equation

$$
\frac{\partial \phi_{i}(t-r / c)}{\partial t}+\frac{1}{2}\left(\frac{\partial \phi_{i}(t-r / c)}{\partial r}\right)^{2}+\frac{p(t-r / c)-p_{\infty}(t)}{\rho_{l, 0}}=0,
$$

where we have used that the derivative of the potential far from the bubble is

$$
\frac{\partial \phi_{\infty}(t)}{\partial t}=-\frac{p_{\infty}(t)-p_{0}}{\rho_{l, 0}} .
$$

The potential generated by the bubble decays with the distance, therefore

$$
\frac{\partial \phi_{i}(t-r / c)}{\partial t}=\frac{\partial \phi_{i}\left(t^{\prime}-r / c\right)}{\partial t} \frac{R_{i}\left(t^{\prime}\right)}{r},
$$


where we have defined a new variable $t^{\prime}=t-\left(r-R_{i}\left(t^{\prime}\right)\right) / c$ in order to relate the solution at time $t$ at distance $r$ with the solution at time $t^{\prime}$ at the bubble interface. Now we can write $\left(\partial \phi_{i}\left(t^{\prime}-R_{i}\left(t^{\prime}\right) / c\right)\right) / \partial t$ as a function of the properties at the interface as

$$
\frac{\partial \phi_{i}\left(t^{\prime}-r / c\right)}{\partial t}=-\frac{1}{2} \dot{R}_{i}^{2}\left(t^{\prime}\right)-\frac{p_{i}\left(t^{\prime}\right)-p_{\infty}\left(t^{\prime}\right)}{\rho_{l, 0}} .
$$

Finally, applying the chain rule, the radial derivative of the potential created by the bubble is

$$
\begin{aligned}
\frac{\partial \phi_{i}(t-r / c)}{\partial r} & =-\frac{1}{c} \frac{\partial \phi_{i}(t-r / c)}{\partial t}-\frac{\phi_{i}(t-r / c)}{r} \\
& =-\frac{\partial \phi_{i}\left(t^{\prime}-r / c\right)}{\partial t} \frac{R_{i}\left(t^{\prime}\right)}{c r}-\phi_{i}\left(t^{\prime}-r / c\right) \frac{R_{i}\left(t^{\prime}\right)}{r^{2}},
\end{aligned}
$$

where we can use the restriction imposed by the boundary condition at time $t^{\prime}$

$$
\frac{\partial \phi_{i}\left(t^{\prime}-r / c\right)}{\partial r}=\dot{R}_{i}\left(t^{\prime}\right)
$$

to express (7.6) as

$$
\frac{\partial \phi_{i}(t-r / c)}{\partial r}=\frac{1}{c} \frac{\partial \phi_{i}}{\partial t}\left(t^{\prime}-r / c\right) \frac{R_{i}\left(t^{\prime}\right)}{r}\left(\frac{R_{i}\left(t^{\prime}\right)}{r}-1\right)+\dot{R}_{i}\left(\frac{R_{i}\left(t^{\prime}\right)}{r}\right)^{2},
$$

which satisfies the boundary condition at $t=t^{\prime}\left(r=R_{i}\left(t^{\prime}\right)\right)$ and tends to the expected expression for the incompressible limit.

Substituting (7.8) into (7.2) the pressure at any time and position around the bubble can be written as a function of the properties at the bubble interface at time $t^{\prime}$ as

$$
\begin{aligned}
p(t-r / c)= & p_{\infty}(t)-\rho_{l, 0} \frac{R_{b}\left(t^{\prime}\right)}{r} \frac{\partial \phi_{i}\left(t^{\prime}-r / c\right)}{\partial t} \\
& -\rho_{l, 0} \frac{1}{2}\left(\frac{\frac{\partial \phi_{i}\left(t^{\prime}-r / c\right)}{\partial t}}{c} \frac{R_{i}\left(t^{\prime}\right)}{r}\left(\frac{R_{i}\left(t^{\prime}\right)}{r}-1\right)+\dot{R}_{i}\left(\frac{R_{i}\left(t^{\prime}\right)}{r}\right)^{2}\right)^{2},
\end{aligned}
$$

where the temporal derivative of the potential at $t^{\prime}-r / c$ is also a function of the properties at $t^{\prime}$ (7.5).

Figure 2 depicts the evolution of the bubble radius for the analytical problem and the current model with and without the correction terms $I^{* *}$ (3.27). Clearly, to reproduce the solution given by the analytical problem, the correction terms are required when the grid size is comparable to the bubble radius. The reason is that when the mesh is refined, the method is able to capture the pressure disturbances induced by the bubble on the surrounding liquid. Therefore, the pressure at the node no longer corresponds to the pressure at infinity, and the prediction of the bubble dynamics requires the correction term. Figure 3 shows how the method is able to correct the errors on the amplitude and collapse time of the bubble oscillation as the size of the bubble becomes of the order of the cell size. As the ratio $R_{0} / \Delta x$ decreases, the influence of the bubble on the length scale of the cell is less important and the correction term is no longer needed.

We also use this model problem to assess the impact of the choice of the kernel function in the limit of a single bubble. In this case, the characteristic length of the support, $h$, that is used to project the gas volume on the grid, determines the accuracy 


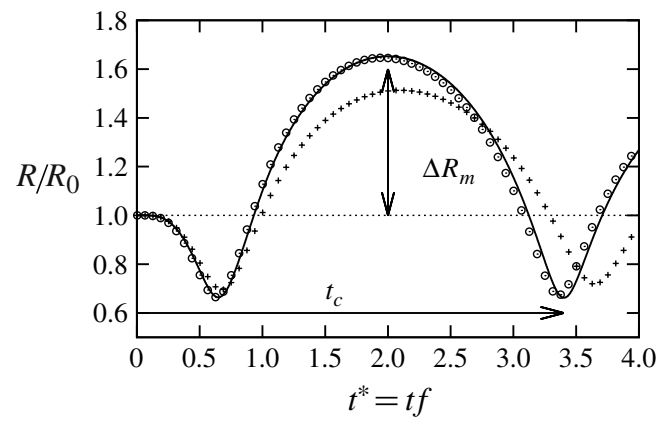

FIGURE 2. Bubble response of a $50 \mu \mathrm{m}$ bubble to a sinusoidal pressure wave of $150 \mathrm{kHz}$ and $2 \mathrm{~atm}$. Bubble radius evolution obtained with the current model with the correction term $I^{* *}(\odot)$, without the correction term $(+)$, and the analytical solution (-). Grid resolution: $\Delta x=4 R_{0}$.
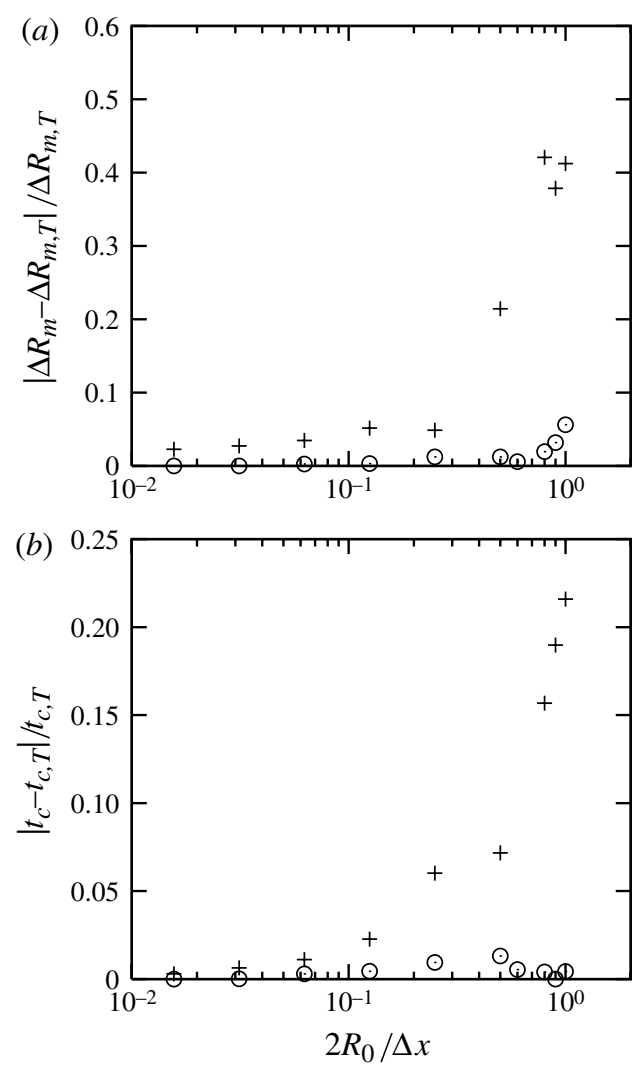

FIGURE 3. (a) Error in the maximum bubble radius and (b) collapse time of the current model with the correction term $I^{* *}(\odot)$ and without it $(+)$ compared with the analytical solution $\left(\Delta R_{m, T}\right.$ and $\left.t_{c, T}\right)$ as a function of the bubble radius. Bubbles forced with a sinusoidal wave of $f=150 \mathrm{kHz}$ and $\Delta p_{\infty}=2 \mathrm{~atm} . \Delta x=2 \times 10^{-4} \mathrm{~m}$.

of the pressure disturbances captured by the current method. We compare in this work the theoretical solution obtained from a one-dimensional model, with the numerical 

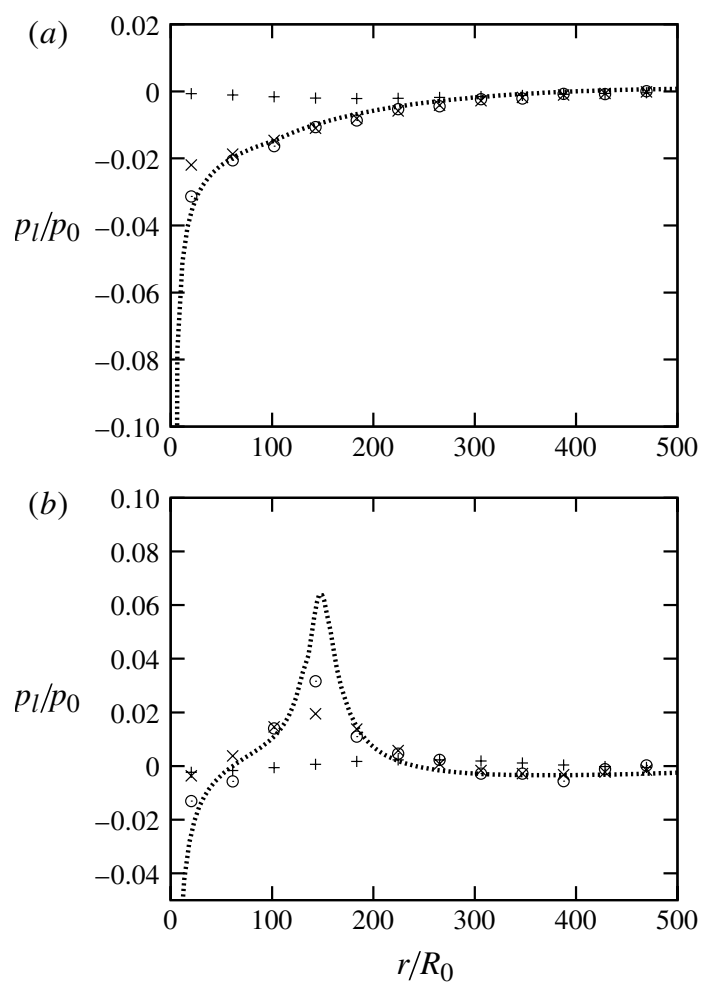

FIGURE 4. Analytical pressure wave $(\ldots$.$) induced by the bubble in the surrounding liquid$ compared to the numerical solution obtained with a discrete delta function $(\odot)$, a Gaussian with a standard deviation equal to the grid size $(x)$ and a Gaussian with a standard deviation equal to two times the grid size (+). The bubble is located at $r=0$. The profiles are shown before the collapse $(a)$ and after it $(b):(a) t f=1.1 ;(b) t f=1.5$.

solutions of the current model using a discrete delta function and a Gaussian with two different lengths of the support ( $\Delta x$ and $2 \Delta x$ respectively). Figure 4 compares the numerical and theoretical solution (given by (7.9)) of the pressure disturbance induced around a bubble of radius $5 \mu \mathrm{m}$ plotted as a function of distance. The method converges to the solution of a single bubble as the length of the support is reduced. The method is able to capture relatively well the waves generated during the bubble collapse. Despite the low spatial resolution of the three-dimensional simulation, when a discrete delta function is used, good agreement is found between the pressure field obtained with the present method and the theoretical pressure. As we increase the length of the support, the method is less accurate in the limit of a single bubble. Thus, we can conclude that the current method consistently obtains both bubble radius evolution and the pressure disturbances induced by the bubble on the surrounding liquid.

\subsection{Bubble cluster model}

In $\$ 3.4$ we have introduced the notion of a surrogate bubble to obtain the term $I^{* *}$ in the bubble dynamic equation. This term has been derived to obtain the analytical solution of single bubble in an infinite medium when it is enclosed within a cell. However, it only gives an approximate solution when there are multiple bubbles in 

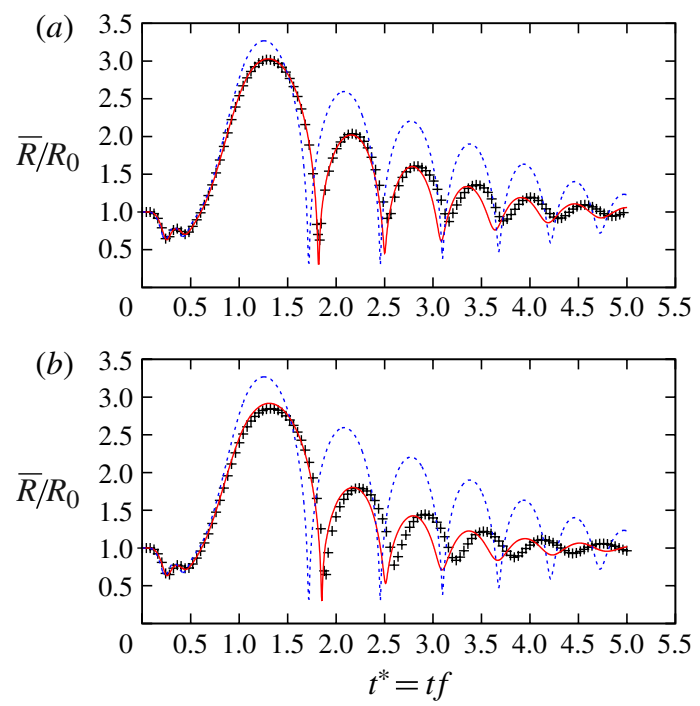

FIGURE 5. (Colour online available at journals.cambridge.org/flm) Averaged bubble radius evolution of a cluster of four bubbles $(a)$ and eight bubbles $(b)$ of radius $10 \mu \mathrm{m}$ exposed to a sinusoidal pressure wave of $150 \mathrm{kHz}$ and $2 \mathrm{~atm}$. The solution obtained for the case where the bubbles are enclosed in a cell (-) matches relatively well that obtained when bubbles are located in different cells $(+)$. The solution of a single isolated bubble is included as reference (- - - ). For the examples tested here, the model used here captures relatively well the main mechanisms of direct bubble-bubble interactions. Domain size: $L_{x}=1200 R_{0}, L_{y}=500 R_{0}$, $L_{z}=250 R_{0}$. Grid spacing $\Delta x=10 R_{0}$ (for -) and $\Delta x=5 R_{0}$ (for + ).

the cell. In this section, we provide two representative examples to examine the accuracy of this approximation. The first one is a cluster of four bubbles of $10 \mu \mathrm{m}$ radius located at the vertices of a square of size $L=10 R_{0}$. An incident sinusoidal wave similar to that of the test case reported in $\S 7.1$ propagates normal to the plane containing the four bubbles. In the second example we consider a cluster of eight bubbles located at the vertices of a cube.

Taking advantage of the convergence of the model in the limit of one bubble per cell, we compare the solution obtained in this limiting case with that obtained when all the bubbles are located inside the same cell. In order to compare the different solutions, we define an averaged bubble radius for the $N$ bubbles contained inside the domain as

$$
\bar{R}=\left(\frac{\sum_{i=1}^{N} V_{i}}{\frac{4}{3} \pi N}\right)^{1 / 3} .
$$

Figure 5 shows that the surrogate bubble model is able to capture the direct bubble-bubble interactions. These effects are clearly observed when we compare the solution with that of a single isolated bubble. Further analyses (not shown in this paper) have shown that the accuracy of the current model also depends on factors such as the spatial bubble distribution and polydispersity inside the cell. In any event, although the degree of accuracy of the current approximation is problem dependent 
and difficult to quantify in general, in all the cases the current model displayed a significant improvement to the solution obtained with the standard version of the Rayleigh-Plesset equation.

\subsection{Highly disperse system}

We next consider the response of a cylindrical, monodisperse, bubble cluster to an incident pressure wave. Previously, it has been argued that for sufficiently low resolution, the solution of the current method should converge to the solution of the continuum model. In the case of monodisperse systems the only condition that must be satisfied is that the kernels for the different bubbles must overlap in order to converge to the solution of the void fraction field used by continuous models. In this section, we compare the solution of the model presented by Ando et al. (2011) with the solution of the current model.

In order to excite a wide range of frequencies, the initial pressure is initialized by imposing a Gaussian planar pressure wave which propagates towards the cluster:

$$
\begin{gathered}
p_{l, 0}(x)=p_{\infty}+\Delta p_{\infty} \exp \left(-0.5\left(\frac{x-x_{0}}{\sigma}\right)^{2}\right), \\
u_{l, 0}(x)=\frac{\Delta p_{\infty}}{\rho_{l} c} \exp \left(-0.5\left(\frac{x-x_{0}}{\sigma}\right)^{2}\right), \\
v_{l, 0}(x)=0 \\
w_{l, 0}(x)=0
\end{gathered}
$$

where $\sigma=0.03 \mathrm{~m}$ and the amplitude of the wave is set to $\Delta p_{\infty}=10 \mathrm{~Pa}$.

The bubble cluster is a cylinder of $R_{c}=0.1 \mathrm{~m}$ of uniformly distributed air bubbles of $100 \mu \mathrm{m}$ radius. The number of bubbles is adjusted to generate a concentration field equal to $0.15 \%$. The value of $L_{z}$ is obtained from (3.33). The dimensions of the two-dimensional domain are $L \times L / 2$, where $L / R_{c}=8$, which is uniformly discretized with a mesh of $800 \times 400$ nodes. The maximum CFL number, defined as $\Delta t_{c} / \Delta x$, is set to 0.1 .

The convergence of the method is depicted in figure 6, where the error at a given point, $\boldsymbol{x}_{0}$, is computed for various grid resolutions as

$$
|e r r|_{L_{1}}=\frac{\int_{0}^{\infty}\left|p_{l}\left(\boldsymbol{x}_{0}, t\right)-p_{l, \text { fine }}\left(\boldsymbol{x}_{0}, t\right)\right| \mathrm{d} t}{\int_{0}^{\infty}\left|p_{l \text { fine }}\left(\boldsymbol{x}_{0}, t\right)\right| \mathrm{d} t},
$$

where the solution of the finest grid $(800 \times 400), p_{l, \text { fine }}$, is taken as an approximation of the exact solution.

Figure 7 compares the temporal evolution of the pressure for the present model and the ensemble-averaged model presented by Ando et al. (2011). Two different locations along the axis of symmetry are sampled in order to measure the amplitude of the reflected and transmitted wave. Good agreement is found between the two solutions for both the reflected and transmitted wave. Again, the discrete delta function displays the best performance in comparison to other kernel functions when compared to the solution of the ensemble-averaged model. However, in this case, the main mechanism influencing the wave propagation, that is the change in the mixture density, is already captured by the current model irrespective of the type of kernel function used. 


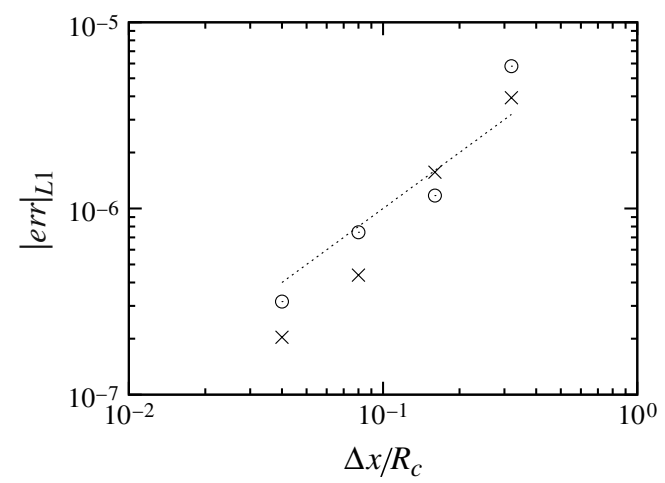

FIGURE 6. $L_{1}$ norm of the error of the temporal evolution of the pressure of the reflected wave $(\odot)$ and transmitted wave $(\times)$. The two points sampled are the same as in figure 7: $\left(-3 R_{c}, 0\right)$ and $\left(3 R_{c}, 0\right)$. $(C F L)_{\max }=0.1$. As reference, the diagonal line represents the convergence of a first-order method.
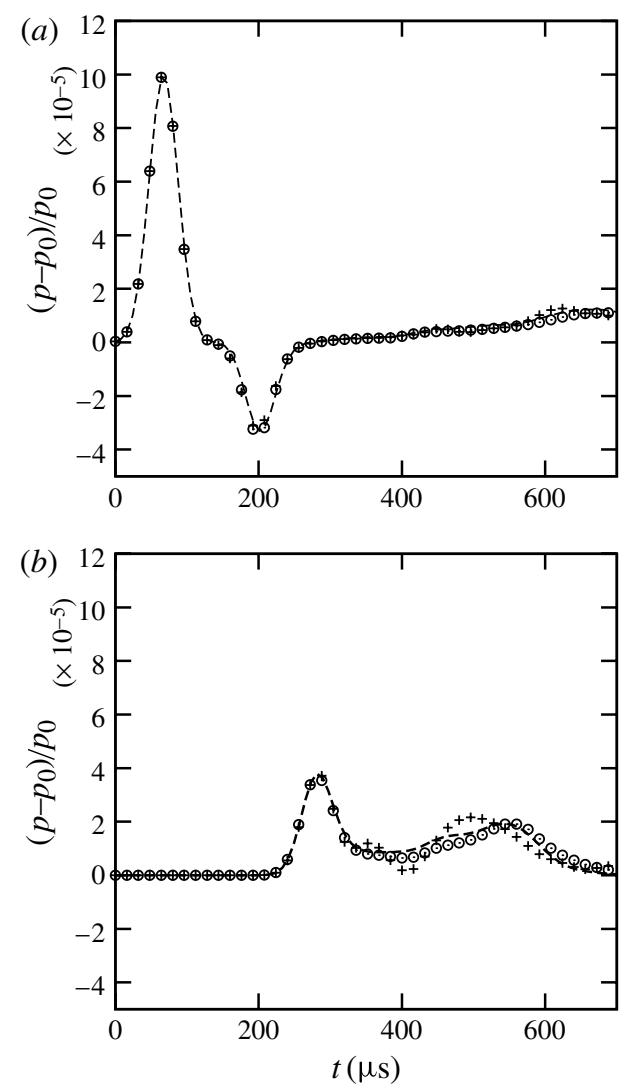

FIGURE 7. Comparison between the present model with a discrete delta function $(\odot)$ and a Gaussian with $h=2 \Delta x(+)$ and the ensemble-averaged model $(-)$ presented by Ando et al. (2011) of the temporal evolution of the liquid pressure at two different locations. Radius of the bubble cluster: $R_{c}=0.05 \mathrm{~m}$. (a) Temporal evolution of the reflected pressure wave at $\left(x / R_{c}, y / R_{c}\right)=(-3,0)$. (b) Temporal evolution of the transmitted pressure wave at $\left(x / R_{c}, y / R_{c}\right)=(3,0)$. 

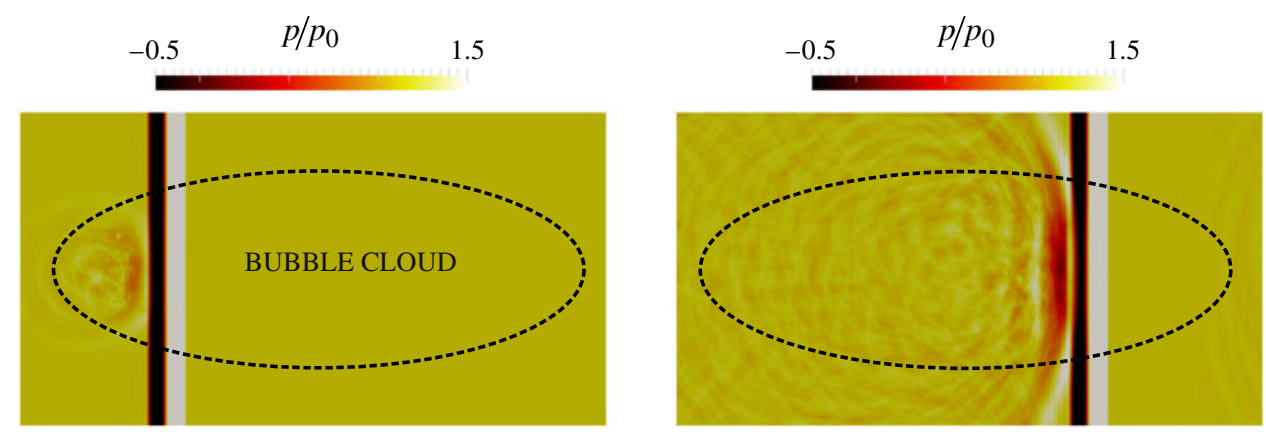

Figure 8. (Colour online) Pressure fields at two different times during the wave propagation. Owing to the low nuclei concentration $\left(500\right.$ nuclei $\left.\mathrm{ml}^{-1}\right)$ the positive region of the wave remains almost unaltered. During the tensile phase, bubbles grow and the void fraction is high enough to induce significant changes on the negative wave. After the expansion, bubbles implode violently inducing secondary pressure waves.

\section{Complex simulations}

\subsection{The effect of bubble nuclei on pressure wave propagation}

As an example of the phenomena that can be captured with the current model, we investigated a two-dimensional ellipsoidal bubble cloud with a major radius of $40 \mathrm{~mm}$ and minor radius of $6.5 \mathrm{~mm}$ exposed to a sinusoidal travelling wave. The positive wave arrives first and the negative part of the wave gives rise to cavitation and bubble implosion. Trying to mimic situations typically encountered in shock-wave lithotripsy investigated by Arora, Ohl \& Lohse (2007), we chose a wave of 2 atm and a wavelength of $6 \mathrm{~mm}$. These values fit relatively well the shape of the tensile part tested in Arora et al. (2007), although the amplitude is about 3 times less intense. The differences in the shock wave arriving first are more pronounced but, as the void fraction is low, we do not expect bubbles to significantly disturb the shape of the wave (this fact has been experimentally verified by Arora et al. 2007).

The ellipsoid is generated by randomly distributing bubbles whose equilibrium radii obey a $\log$-normal distribution with $\bar{R}=5 \mu \mathrm{m}$ and $\sigma=0.7$. Two different nuclei concentrations are tested: 50 and 500 nuclei $\mathrm{ml}^{-1}$.

Figure 8 depicts the pressure fields at two different times. Large void fractions are only achieved when the negative part of the wave arrives. Thus, the positive part of the wave remains essentially unaltered by the presence of the bubbles whereas the structure of the negative part of the wave significantly changes as it propagates throughout the cloud. The presence of bubble nuclei promotes secondary waves after the tail phase. The pressure waves induced during bubble collapse are also observed and responsible for the complex system of pressure disturbances observed in the liquid.

Figure 9 depicts the temporal evolution of the liquid pressure at the centre of the bubble cloud for three different bubble nuclei concentrations. The increase of the bubble nuclei concentration induces significant changes in the structure of the tensile phase. The structure of the waves induced by the presence of the bubble nuclei is similar to that observed by Arora et al. (2007): the strength and frequency of the secondary waves increases with the concentration of bubble nuclei. 


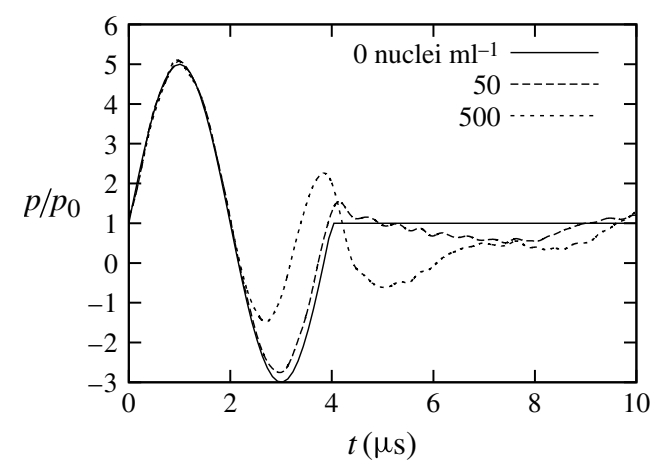

FIGURE 9. Liquid pressure evolution at the centre of the bubble cloud for three different concentrations of bubble nuclei: 0,50 and 500 nuclei $\mathrm{ml}^{-1}$. The presence of bubble nuclei induces a positive wave whose amplitude increases with the concentration of bubbles. The structure of this wave promoted by the presence of bubbles is also similar to that experimentally observed by Arora et al. (2007).

\subsection{Response of a bubble cluster to a sinusoidal pressure wave}

The last example consists of the response of a cylindrical bubble cloud to a sinusoidal pulse. In this case, the negative part of the wave arrives first, which significantly strengthens the violence of the collapse of the bubbles. The amplitude of the wave is set to $\Delta p / p_{0}=1.8$ and the wavelength is $\lambda / R_{c}=2, R_{c}$ being the radius of the cluster. The bubble cluster is generated by random location of bubble nuclei in a circular region. The bubble radius distribution of the nuclei obeys a log-normal distribution with $\sigma=0.7$ and $\bar{R} / R_{c}=10^{-4} \ll \lambda$. The averaged void fraction is $10^{-5} \mathrm{~m}^{3}$ gas $\mathrm{m}^{-3}$. To avoid reflections, the size of the two-dimensional domain is $\left[L_{x} / R_{c}, L_{y} / R_{c}\right]=[20,10]$. In order to accurately predict the conditions inside the cluster, the mesh is concentrated at the centre of the bubble cloud where the spatial resolution is maximum.

As depicted in figure 10, the collapse of the bubbles generates large pressures inside the cluster that induce a very complex response of the fluid. Figure 11 shows the evolution of the pressure at the centre of the cloud for different spatial resolutions. As we refine we are able to better capture the pressure wave emitted by the collapse of the bubbles. The low-frequency signal can be relatively well captured for low levels of refinement, where the solution of the current model and that of ensemble-averaged models are expected to converge.

The simulation included here required $24 \mathrm{~h}$ at the highest level of resolution on a dual-core AMD Opteron Processor 270 machine at $1990 \mathrm{MHz}$. Thus, the current model is suitable to investigate complex situations in polydisperse mixtures, obtaining a reasonable estimation not only of the low-frequency structures provided by ensembleaveraged models, but also details about the peak pressures generated by the collapse of single bubbles in the cluster.

\section{Conclusions}

This work presents a new model to simulate bubbly flows. Phenomena such as liquid compressibility, heat and mass transfer across the bubble interface, bubble motion, bubble-bubble interaction and viscous effects in the liquid have been modelled. The model, based on the volume-averaged Navier-Stokes equations, is 


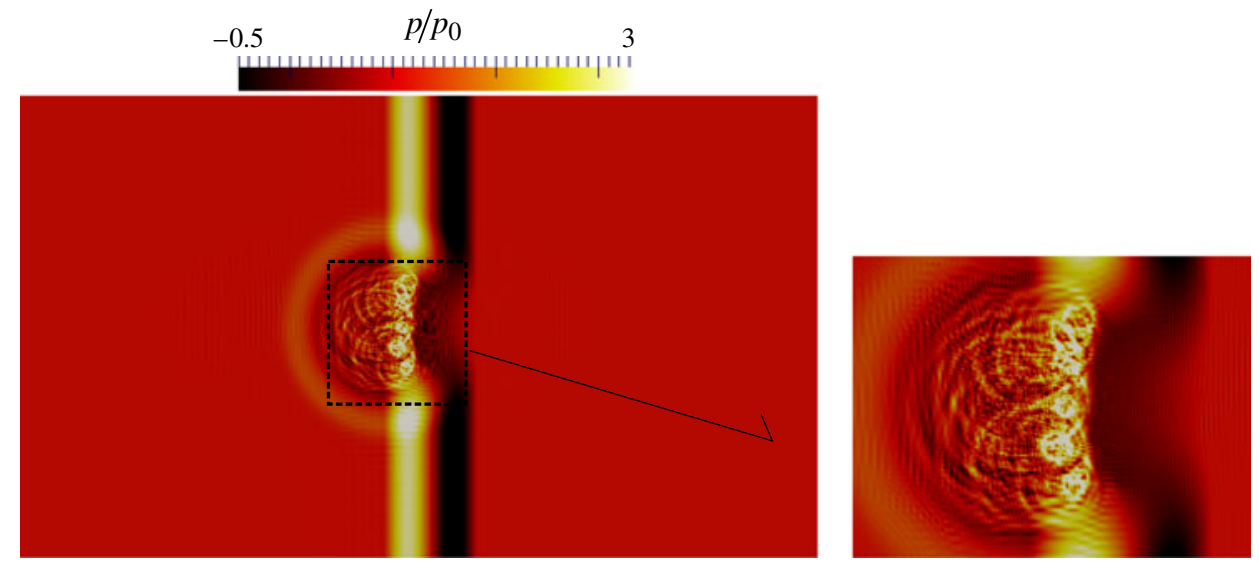

FIgURE 10. (Colour online) Non-dimensional pressure field during the collapse of a cylindrical bubble cloud excited with a single sinusoidal pulse. Both the reflected wave and the waves emitted by single bubbles are captured.

designed to reproduce the bubble oscillations of spherical bubbles that are not necessarily much smaller than the grid size.

By decomposing the potential inside the cell into a background potential and the potential induced by each of the bubbles contained in it, an extended Rayleigh-Plesset equation is derived. This equation accounts for the presence of other bubbles, liquid compressibility effects and the finite size of the cell. The method is able to reproduce not only the radial oscillation of bubbles whose size is of the order of the grid size, but it also captures the pressure field generated around the bubble using only local information in the vicinity of the bubble. The results become independent of the mesh in the limit of one bubble per cell, where the model is verified against the theoretical solution for a single bubble.

At the other extreme, the current model converges to ensemble-averaged models for low spatial resolutions. Thus, the solution of the current model for low spatial resolutions can be interpreted as an approximation of the averaged solution provided by ensemble-averaged models. However the computational effort required for the current models is significantly reduced compared to ensemble-averaged models. Therefore, the current model seems suitable to numerically investigate complex problems where the computational effort of current ensemble-averaged models is prohibitive.

At higher spatial resolution, the model provides information about the instantaneous local pressures associated with individual bubbles. This represents an advantage over ensemble-averaged models which cannot represent the instantaneous pressures reached in an individual realization.

An example in the context of lithotripsy is shown as a proof of the potential of the code to investigate real problems involving large numbers of bubbles and wave propagation. Current results are in qualitative agreement with the experimental observations by Arora et al. (2007), showing that the presence of bubble nuclei induces a positive wave whose amplitude increases with the concentration of bubbles.

The authors would like thank $\mathrm{Dr} \mathrm{K}$. Ando for his generous assistance in making the comparisons with his ensemble-averaged models. We would also like 

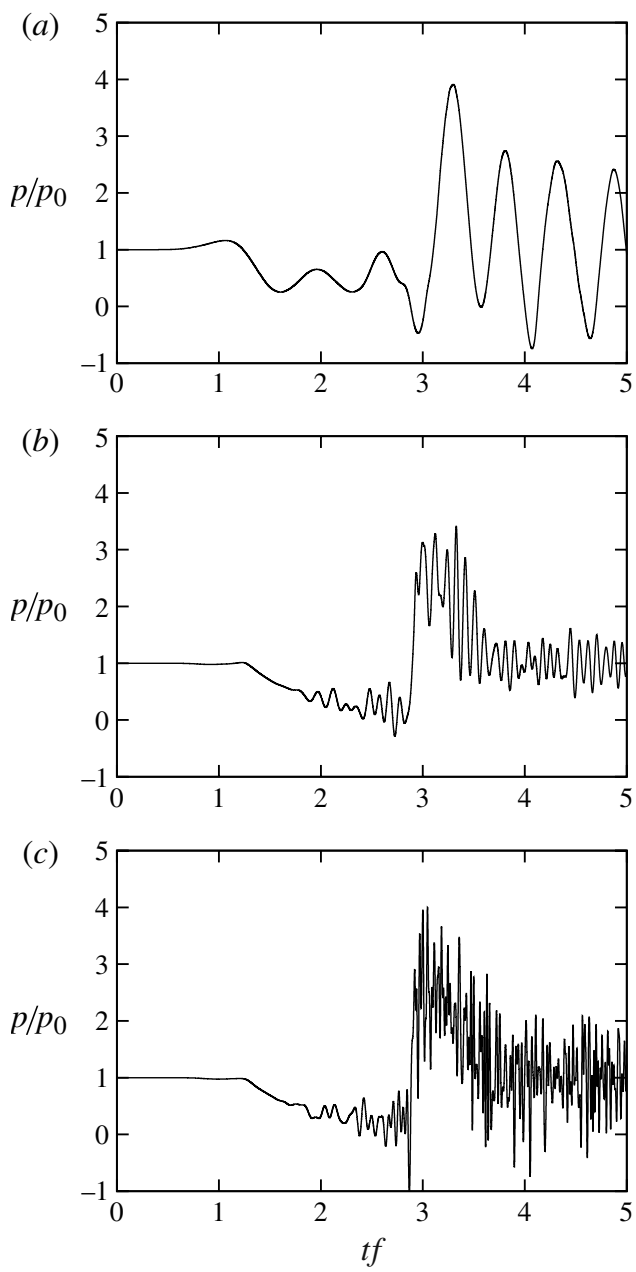

FIGURE 11. Temporal evolution of the liquid pressure at the centre of the bubble cloud of figure 10 for different spatial resolutions: $\Delta x_{\min } / R_{c}=0.12(a), 0.02(b), 0.005(c)$.

to thank Professor C. Brennen for his helpful suggestions. The first author gratefully acknowledges the support of the Spanish Ministry of Education (grant E-28-20090540927). This work was supported in part by the Office of Naval Research under grant N00014-06-1-0730.

\section{Appendix. The bubble dynamic model}

\section{A.1. Basic equations}

The goal of this appendix is to provide an equation for the bubble motion that takes into account the potential distributions inside the cell. RP-like equations for compressible flows can be obtained by solving a system of two equations (Gilmore 1952; Keller \& Miksis 1980). Under the assumption of potential flow and taking into account liquid compressibility, the potential of a fluid obeys the wave equation

$$
\frac{1}{c^{2}} \frac{\partial^{2} \phi}{\partial t^{2}}=\nabla^{2} \phi
$$


The transient Bernoulli equation can be applied for every two different points of the fluid irrespective of their location. Thus, the temporal derivative of the total potential at the location of the bubble $i$ is

$$
\frac{\partial \phi}{\partial t}+\frac{1}{2}\left|\boldsymbol{u}_{l}\right|^{2}+\int_{p_{0}}^{p_{i}} \frac{\mathrm{d} p}{\rho}=0 .
$$

\section{A.2. Boundary conditions}

The system of equations (A 1)-(A 2) has a unique solution given appropriate initial and boundary conditions. At the interface of each bubble, in the absence of relative motion, the potential must obey the boundary condition

$$
\dot{R}_{i}=\frac{\partial \phi_{T}\left(R_{i}, t\right)}{\partial r},
$$

where $\boldsymbol{r}$ represents the radial coordinate of a system of coordinates centred at the location of the $i$ th bubble. The derivation of the RP equation rests upon the assumption that bubble motion remains spherical at every moment. For that reason, to deduce the new equation, it is convenient to express the global potentials decomposed as an incoming and an outgoing spherical wave.

Equation (A 3) requires the radial derivatives of the potentials. In the next sections, we obtain expressions for these derivatives.

\section{A.2.1. Potential created by the background flow}

The presence of an external source is modelled by a uniform potential inside the cell; therefore,

$$
\frac{\partial \phi_{\infty}\left(R_{i}\right)}{\partial r}=0 .
$$

The temporal derivative of the potential at infinity must be known. The derivation of its expression as a function of the averaged values in the cell can be found in $\S 3.4$.

\section{A.2.2. Potential created by the bubble $i$}

The potential $\phi_{i}$ induced by every bubble can be seen as an outward wave emitted from the interface of the $i$ th bubble

$$
\phi_{i}=\frac{f_{i}(t-r / c)}{r_{i}},
$$

whose radial derivative evaluated at the interface is

$$
\frac{\partial \phi_{i}\left(R_{i}\right)}{\partial r}=-\frac{1}{c} \frac{\partial \phi_{i}\left(R_{i}\right)}{\partial t}-\frac{\phi_{i}\left(R_{i}\right)}{R_{i}} .
$$

\section{A.2.3. Potential created by the rest of the bubbles $j$ around the bubble $i$}

The incoming potential created by the bubble $j$ at the location of the bubble $i$ is modelled as an incoming wave

$$
\phi_{j}=\frac{f_{j}\left(t-r_{j} / c\right)}{r_{j}}=\frac{f_{j}\left(t-d_{i j} / c+r_{i} / c\right)}{d_{i j}-r_{i}},
$$

where $r_{j}$ represents the system of coordinates located at the centre of the bubble $j, r_{i}$ the radial coordinate in a system of reference located at the centre of the bubble $i$ and $d_{i j}$ is the distance in absolute value between the two bubbles.

Because $\phi_{j}\left(r=R_{i}\right)$ is not uniform along the interface of the bubble $i$, further modelling is required in order to find an expression for the influence of the potential 

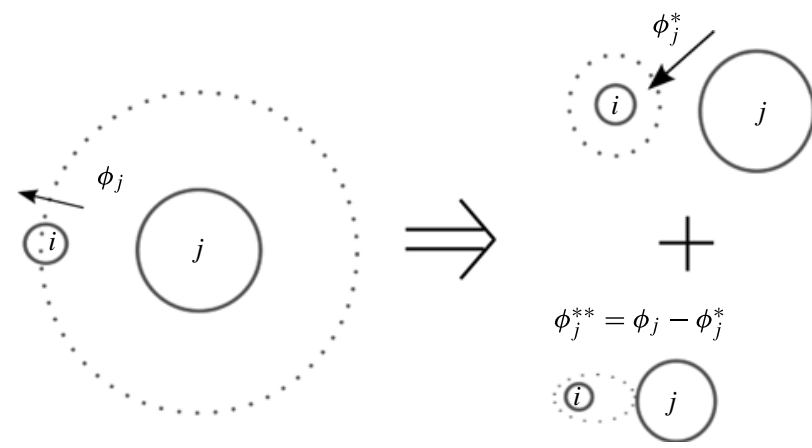

FIGURE 12. Potential decomposition of the potential induced by the bubble $j$ on the bubble $i$. $\phi_{j}^{*}\left(r_{i}\right)$ represents a spherical incoming wave for the $j$ th bubble whereas $\phi_{j}^{* *}\left(r_{i}\right)$ is defined as the difference between the outgoing potential for the $i$ th bubble and $\phi_{j}^{*}\left(r_{i}\right)$.

of the bubble $j$ on $i$. This potential is split into a radial part, $\phi_{j}^{*}$, that we model in the surroundings of the bubble $i$ as an equivalent incoming spherical wave whose potential is equal to that induced by $j$ at the centre of the bubble $i$, and a potential $\phi_{j}^{* *}$, which is defined as (see figure 12)

$$
\begin{gathered}
\phi_{j}^{*}\left(r_{i}\right)=\frac{f_{j}^{*}\left(t+r_{i} / c\right)}{r_{i}}, \\
\phi_{j}^{* *}\left(r_{i}\right)=\phi_{j}-\phi_{j}^{*}\left(r_{i}\right)=\frac{f_{j}\left(t-d_{i j} / c+r_{i} / c\right)}{d_{i j}-r_{i}}-\frac{f_{j}^{*}\left(t+r_{i} / c\right)}{r_{i}},
\end{gathered}
$$

where, by definition, we impose the following condition:

$$
\begin{gathered}
\phi_{j}^{*}\left(r_{i}=0\right)=\frac{f_{j}^{*}(t)}{r_{i}}=\frac{f_{j}\left(t-d_{i j} / c\right)}{d_{i j}}, \\
\phi_{j}^{* *}\left(r_{i}=0\right)=0 .
\end{gathered}
$$

Note that $\phi_{j}^{*}\left(r_{i}=0\right)$, and therefore $f_{j}^{*}(t)$, is directly obtained from the potential emitted by the $j$ th bubble evaluated at the position of the $i$ th bubble. As $\phi_{j}^{*}$ is symmetric and continuous at $r=0$ all its derivatives are 0 at the bubble centre. Therefore, applying a Taylor series expansion, the potential in the surroundings of the bubble centre can be accurately approximated by

$$
\phi_{j}^{*}\left(r_{i}\right) \approx \frac{f_{j}\left(t-d_{i j} / c\right)}{d_{i j}},
$$

whereas the expansion for $\phi_{j}^{* *}$ is

$$
\phi_{j}^{* *}\left(r_{i}\right)=\frac{\partial \phi_{j}^{* *}(0)}{\partial r} r_{i}+\frac{1}{2} \frac{\partial^{2} \phi_{j}^{* *}(0)}{\partial r^{2}} r_{i}^{2}+\cdots
$$

The first terms can be evaluated using (A 9)-(A 10)

$$
\begin{gathered}
\frac{\partial \phi_{j}^{* *}(0)}{\partial r}=\frac{\partial}{\partial r}\left(\frac{f_{j}\left(t-d_{i j} / c\right)}{d_{i j}}\right)-\frac{\partial}{\partial r}\left(\frac{f_{j}^{*}(t)}{r_{i}}\right)_{r=0}=\frac{\phi(0)}{d_{i j}}, \\
\frac{\partial^{2} \phi_{j}^{* *}(0)}{\partial r^{2}}=\frac{\phi_{j}(0)}{d_{i j}^{2}}, \ldots
\end{gathered}
$$


Thus, for $r_{i} \ll d_{i j}$, we can obtain an accurate representation of the potential in the surroundings of the bubble $i$ by

$$
\phi_{j}\left(r_{i}\right)=\frac{f_{j}\left(t-d_{i j} / c\right)}{d_{i j}}\left(1+\frac{r_{i}}{d_{i j}}+\frac{1}{2}\left(\frac{r_{i}}{d_{i j}}\right)^{2}+\cdots\right),
$$

whose temporal derivative evaluated at the bubble interface is

$$
\frac{\partial \phi_{j}\left(R_{i}\right)}{\partial t}=\frac{1}{d_{i j}} \frac{\partial f_{j}\left(t-d_{i j} / c\right)}{\partial t} S+\frac{f_{j}\left(t-d_{i j} / c\right)}{d_{i j}} \frac{\partial S}{\partial t},
$$

where

$$
S=1+\frac{R_{i}}{d_{i j}}+\frac{1}{2}\left(\frac{R_{i}}{d_{i j}}\right)^{2}+\cdots .
$$

From the practical point of view, it is more convenient to express all the functions in terms of $t^{\prime}=t-\left(d_{i j}-R_{j}\left(t^{\prime}\right)\right) / c$, which represents the delay in time from the emission of the potential from the bubble $j$ to the location of the bubble $i$. To take the temporal derivative of $f_{j}$ we apply the chain rule. For any generic function $F$ depending on $t^{\prime}$ we have

$$
\frac{\partial F}{\partial t}=\frac{\partial F}{\partial t^{\prime}} \frac{\partial t^{\prime}}{\partial t}=\frac{\partial F}{\partial t^{\prime}}\left(1+\frac{\dot{R}_{j}\left(t^{\prime}\right)}{c}\right) .
$$

Thus,

$$
\frac{1}{d_{i j}} \frac{\partial f_{j}\left(t-d_{i j} / c\right)}{\partial t}=\left(1+\frac{\dot{R}_{j}\left(t^{\prime}\right)}{c}\right) \frac{1}{d_{i j}} \frac{\partial f_{j}\left(t^{\prime}-R_{j}\left(t^{\prime}\right) / c\right)}{\partial t^{\prime}} .
$$

Using (A 20), the temporal derivative of the potential $j$ evaluated at the interface of the bubble $i$ (A 17) is evaluated as

$$
\begin{aligned}
\frac{\partial \phi_{j}\left(R_{i}\right)}{\partial t}= & \frac{R_{j}\left(t^{\prime}\right)}{d_{i j}} \frac{\partial \phi_{j}\left(t^{\prime}-R_{j}\left(t^{\prime}\right) / c\right)}{\partial t^{\prime}}\left(1+\frac{\dot{R}_{j}\left(t^{\prime}\right)}{c}\right) S \\
& +\frac{R_{j}\left(t^{\prime}\right)}{d_{i j}} \phi_{j}\left(t^{\prime}-R_{j}\left(t^{\prime}\right) / c\right) \frac{\partial S}{\partial t^{\prime}}\left(1+\frac{\dot{R}_{j}\left(t^{\prime}\right)}{c}\right) .
\end{aligned}
$$

For the sake of simplicity, for further development we only consider the first term in the Taylor expansion $\left(S=1\left(\partial S / \partial t^{\prime}\right)=0\right)$, which means that the interaction between two bubbles is given by evaluation of $\partial \phi_{j} / \partial t$ at the centre of the $i$ th bubble. Note that in those situations where this term governs the bubble dynamics, some of the assumptions made here (e.g. sphericity) could not be applicable. Thus, being aware of the uncertainty of the model in these situations, the zeroth-order approximation will be usually enough to qualitatively investigate the effect of the interactions.

\section{A.2.4. Derivation of the modified Rayleigh-Plesset equation}

Once the expressions for the different potentials have been obtained it is possible to derive a generalized Rayleigh-Plesset equation by applying the boundary condition

$$
\dot{R}_{i}=\frac{\partial \phi_{\infty}\left(R_{i}\right)}{\partial r}+\frac{\partial \phi_{i}\left(R_{i}\right)}{\partial r}+\sum_{j \neq i}^{N} \frac{\partial \phi_{j}\left(R_{i}\right)}{\partial r} .
$$

Considering that the background flow induces a uniform potential and neglecting the radial component of the potential induced by the bubble $j$, we can multiply (A 22) 
by $c R_{i}$ to obtain

$$
c R_{i} \dot{R}_{i}=-R_{i} \frac{\partial \phi_{i}\left(R_{i}\right)}{\partial t}-c \phi_{i}\left(R_{i}\right),
$$

where (A 6) has been used to replace the radial derivative of the potential.

On the other hand, we can apply the Bernoulli equation from the bubble interface to the reference state to obtain

$$
\frac{\partial \phi_{i}\left(R_{i}\right)}{\partial t}=-\frac{\dot{R}_{i}^{2}}{2}-H_{i}-\sum_{j \neq i}^{N} \frac{\partial \phi_{j}\left(R_{i}\right)}{\partial t}-\frac{\partial \phi_{\infty}}{\partial t} .
$$

Substituting (A 24) into (A 23):

$$
c R_{i} \dot{R}_{i}=\frac{\dot{R}_{i}^{2}}{2} R_{i}+H_{i} R_{i}+R_{i} \frac{\partial \phi_{\infty}}{\partial t}-c \phi_{i}\left(R_{i}\right)+R_{i} \sum_{j \neq i}^{N} \frac{\partial \phi_{j}\left(R_{i}\right)}{\partial t} .
$$

Now we rewrite (A 25) as

$$
c R_{i} \dot{R}_{i}=\frac{1}{2} R_{i}(t) \dot{R}^{2}+R_{i}(t) H_{i}(t)-c \phi_{i}\left(r=R_{i}\right)+F_{\infty}+I,
$$

where $F_{\infty}$ represents the terms appearing due to the forcing induced by the background field

$$
F_{\infty}=R_{i} \frac{\partial \phi_{\infty}}{\partial t},
$$

and $I$ represents the terms appearing due to the presence of the rest of the bubbles contained inside the cell

$$
I=R_{i} \sum_{j \neq i}^{N} \frac{\partial \phi_{j}\left(R_{i}\right)}{\partial t} .
$$

The temporal derivative of (A 26) is

$$
\begin{aligned}
c \dot{R}_{i}^{2}+c R_{i} \ddot{R}_{i}= & \frac{1}{2} \dot{R}_{i}^{3}(t)+R_{i}(t) \ddot{R}_{i}(t) \dot{R}_{i}(t)+\dot{R}_{i} H_{i}(t)+R_{i} \dot{H}_{i} \\
& -c\left(1-\frac{\dot{R}_{i}}{c}\right) \frac{1}{R_{i}} \frac{\partial f_{i}\left(t-R_{i} / c\right)}{\partial t}+\frac{c \phi_{i}(t)}{R_{i}} \dot{R}_{i}+\frac{\partial F}{\partial t}+\frac{\partial I}{\partial t},
\end{aligned}
$$

where we have applied

$$
\frac{\partial \phi_{i}\left(R_{i}\right)}{\partial t}=\left(1-\frac{\dot{R}_{i}}{c}\right) \frac{1}{R_{i}} \frac{\partial f_{i}\left(t-R_{i} / c\right)}{\partial t}-\frac{\phi_{i}(t)}{R_{i}} \dot{R}_{i} .
$$

From (A 26) we know that

$$
c \phi_{i}\left(r=R_{i}\right)=\frac{1}{2} R_{i}(t) \dot{R}_{i}^{2}+R_{i}(t) H_{i}(t)+F+I-c R_{i} \dot{R}_{i} .
$$

Equations (A 31)-(A 24) can be used to express (A 29) as

$$
\begin{aligned}
R_{i} \ddot{R}_{i}\left(1-\frac{\dot{R}_{i}}{c}\right)+\frac{3}{2} \dot{R}_{i}^{2}\left(1-\frac{\dot{R}_{i}}{3 c}\right)= & H_{i}\left(1+\frac{\dot{R}_{i}}{c}\right)+\frac{R_{i} \dot{H}_{i}}{c} \\
& +\frac{1}{c} \frac{\partial F}{\partial t}+\frac{\dot{R}_{i}}{c R_{i}} F+\left(1-\frac{\dot{R}_{i}}{c}\right) \frac{\partial \phi_{\infty}}{\partial t} \\
& +\frac{1}{c} \frac{\partial I}{\partial t}+\frac{\dot{R}_{i}}{c R_{i}} I+\left(1-\frac{\dot{R}_{i}}{c}\right) \sum_{j \neq i}^{N} \frac{\partial \phi_{j}\left(R_{i}\right)}{\partial t}
\end{aligned}
$$


Given (A 27), $\partial F_{\infty} / \partial t$ can be obtained as

$$
\frac{\partial F_{\infty}}{\partial t}=\dot{R}_{i} \frac{\partial \phi_{\infty}}{\partial t}+R_{i} \frac{\partial^{2} \phi_{\infty}}{\partial t^{2}} .
$$

Thus, the sum of the terms involving $F_{\infty}$ and $\partial F_{\infty} / \partial t$ in (A 32) can be written as

$$
F^{*}=\frac{1}{c} \frac{\partial F_{\infty}}{\partial t}+\frac{\dot{R}_{i}}{c R_{i}} F_{\infty}+\left(1-\frac{\dot{R}_{i}}{c}\right) \frac{\partial \phi_{\infty}}{\partial t}=\frac{\partial \phi_{\infty}}{\partial t}\left(1+\frac{\dot{R}_{i}}{c}\right)+\frac{R_{i}}{c} \frac{\partial^{2} \phi_{\infty}}{\partial t^{2}} .
$$

On the other hand, the sum of all the terms related to the presence of other bubbles is

$$
I^{*}=\frac{1}{c} \frac{\partial I}{\partial t}+\frac{\dot{R}_{i}}{c R_{i}} I+\left(1-\frac{\dot{R}_{i}}{c}\right) \sum_{j \neq i}^{N} \frac{\partial \phi_{j}\left(R_{i}\right)}{\partial t},
$$

where the first term is

$$
\frac{1}{c} \frac{\partial I}{\partial t}=\frac{\dot{R}_{i}}{c} \sum_{j \neq i}^{N} \frac{\partial \phi_{j}\left(R_{i}\right)}{\partial t}+\sum_{j \neq i}^{N}\left[\frac{R_{i}}{d_{i j}} \frac{\partial \phi_{j}\left(R_{i}\right)}{\partial t}\right] .
$$

This equation depends on the potential and its derivative of bubble $j$ at $r_{i}=R_{i}$. These terms can be written as a function of the time $t^{\prime}=t-\left(d_{i j}-R_{j}\left(t^{\prime}\right)\right) / c$. This means that there is a certain lag between the emission of the wave from the bubble $j$ and the reception of the information at the location of the $i$ th bubble.

Applying the chain rule to the second term on the right-hand side and substituting, $I^{*}$ can be then written as

$$
I^{*}=\sum_{j \neq i}^{N}\left[\left(1+\frac{\dot{R}_{i}}{c}\right) \frac{\partial \phi_{j}\left(R_{i}\right)}{\partial t}\right]+\frac{R_{i}}{c} \sum_{j \neq i}^{N}\left[\left(1+\frac{\dot{R}_{j}\left(t^{\prime}\right)}{c}\right) \frac{\partial}{\partial t^{\prime}}\left(\frac{\partial \phi_{j}\left(R_{i}\right)}{\partial t}\right)\right] .
$$

Equation (A 21) provides $\partial \phi_{j}\left(R_{i}\right) / \partial t$ in terms of $t^{\prime}$. Its derivative is

$$
\frac{\partial}{\partial t^{\prime}}\left(\frac{\partial \phi_{j}(0)}{\partial t}\right)=\left[\dot{R}_{j}\left(t^{\prime}\right) d_{i j} \frac{\partial \phi_{j}\left(t^{\prime}-R_{j}\left(t^{\prime}\right) / c\right)}{\partial t^{\prime}}+\frac{R_{j}\left(t^{\prime}\right)}{d_{i j}} \frac{\partial^{2} \phi_{j}\left(t^{\prime}-R_{j}\left(t^{\prime}\right) / c\right)}{\partial t^{\prime 2}}\right] .
$$

Analogously to (A 30), the temporal derivative of the potential at the interface of the bubble $j$ is

$$
\frac{\partial^{2} \phi_{j}\left(t^{\prime}-R_{j}\left(t^{\prime}\right) / c\right)}{\partial t^{\prime 2}}=\left(1-\frac{\dot{R}_{j}}{c}\right) \frac{\partial^{2} \phi_{j}\left(t^{\prime}-R_{j}\left(t^{\prime}\right) / c\right)}{t^{\prime 2}}-\frac{\partial \phi_{j}\left(t^{\prime}-R_{j}\left(t^{\prime}\right) / c\right)}{\partial t^{\prime}} \frac{\dot{R}_{j}}{R_{j}} .
$$

The second derivative of the potential evaluated at the interface can be worked out by differentiating the Bernoulli equation

$$
\frac{\partial^{2} \phi_{j}\left(t^{\prime}-R_{j}\left(t^{\prime}\right) / c\right)}{\partial t^{\prime 2}}=-\dot{R}_{j} \ddot{R}_{j}-\dot{H}_{j}-\sum_{k \neq j}^{N} \frac{\partial \phi_{k}\left(t^{\prime}-R_{j}\left(t^{\prime}\right) / c\right)}{\partial t}-\frac{\partial^{2} \phi_{\infty}}{\partial t^{\prime 2}} .
$$

Then, (A 38) can be finally written as

$$
\frac{\partial}{\partial t^{\prime}}\left(\frac{\partial \phi_{j}\left(R_{i}\right)}{\partial t}\right)=-\frac{R_{j}\left(t^{\prime}\right)}{d_{i j}}\left(1-\frac{\dot{R}_{j}}{c}\right)\left(\dot{R}_{j} \ddot{R}_{j}+\dot{H}_{j}+\sum_{k \neq j}^{N} \frac{\partial \phi_{k}\left(t^{\prime}-R_{j}\left(t^{\prime}\right) / c\right)}{\partial t}+\frac{\partial^{2} \phi_{\infty}}{\partial t^{\prime 2}}\right) .
$$


In addition, the potential and its temporal derivative at $r_{i}=0$ can be written in terms of $t^{\prime}$ as

$$
\begin{gathered}
\phi_{j}\left(R_{i}\right)=\frac{R_{j}\left(t^{\prime}\right)}{d_{i j}} \phi_{j}\left(t^{\prime}-R_{j}\left(t^{\prime}\right)\right), \\
\frac{\partial \phi_{j}\left(R_{i}\right)}{\partial t}=\frac{R_{j}\left(t^{\prime}\right)}{d_{i j}} \frac{\partial \phi_{j}\left(t^{\prime}-R_{j}\left(t^{\prime}\right)\right)}{\partial t^{\prime}} .
\end{gathered}
$$

After deducing expressions for all the terms in (A 32), one can pose the following explicit system of equations to work out the bubble dynamics of every individual bubble:

$$
\begin{gathered}
\ddot{R}_{i}=\left[H_{i}\left(1+\frac{\dot{R}_{i}}{c}\right)+\frac{R_{i} \dot{H}_{i}}{c}+F^{*}+I^{*}-\frac{3}{2} \dot{R}_{i}^{2}\left(1-\frac{\dot{R}_{i}}{3 c}\right)\right]\left(R_{i}\left(1-\frac{\dot{R}_{i}}{c}\right)\right)^{-1}, \\
F^{*}=\frac{\partial \phi_{\infty}}{\partial t}\left(1+\frac{\dot{R}_{i}}{c}\right)+\frac{R_{i}}{c} \frac{\partial^{2} \phi_{\infty}}{\partial t^{2}} \\
I^{*}=\sum_{j \neq i}^{N}\left[\left(1+\frac{\dot{R}_{i}}{c}\right) \frac{\partial \phi_{j}\left(R_{i}\right)}{\partial t}\right] \\
+\frac{R_{i}}{c} \sum_{j \neq i}^{N}\left[\left(1+\frac{\dot{R}_{j}\left(t^{\prime}\right)}{c}\right)\left(1-\frac{\dot{R}_{j}}{c}\right) \frac{\partial^{2}\left(t^{\prime}-R_{j}\left(t^{\prime}\right) / c\right) \phi_{j}}{\partial t^{\prime 2}}\right] \\
-\sum_{j \neq i}^{N}\left[\left(1+\frac{\dot{R}_{j}\left(t^{\prime}\right)}{c}\right) \frac{\partial \phi_{j}\left(t^{\prime}-R_{j}\left(t^{\prime}\right) / c\right)}{\partial t^{\prime}} \frac{R_{i}}{R_{j}} \frac{\dot{R}_{j}}{c}\right] \\
\frac{\partial^{2} \phi_{i}\left(R_{i}\right)}{\partial t^{2}}=-\left(\dot{R}_{i} \ddot{R}_{i}+\dot{H}_{i}+\sum_{j \neq i}^{N} \frac{\partial^{2} \phi_{j}\left(R_{i}\right)}{\partial t^{2}}+\frac{\partial^{2} \phi_{\infty}}{\partial t^{2}}\right) . \\
\frac{\partial^{2} \phi_{j}\left(R_{i}\right)}{\partial t^{2}}=\frac{R_{j}\left(t^{\prime}\right)}{d_{i j}} \frac{\partial^{2} \phi_{j}\left(t^{\prime}-R_{j} / c\right)}{\partial t^{2}} .
\end{gathered}
$$

Provided proper initial conditions for the bubble radius and the potential and its respective derivatives are used, the system of equations above can be integrated in time.

\section{A.2.5. Remarks}

(i) For small density variations, a good estimation of $H_{i}$ is

$$
H_{i}=\frac{p_{i}-p_{0}}{\rho_{l, 0}} .
$$

We also remark that $p_{l}\left(\boldsymbol{x}=\boldsymbol{x}_{i}^{\prime}\right)$ denotes the pressure at the liquid interface of bubble $i$, which can be related to the bubble pressure by applying a momentum balance at the interface of bubble $i$

$$
p_{i}=p_{b, i}-\frac{4 \mu_{l} \dot{R}}{R}-\frac{2 \sigma}{R_{i}}-J^{2}\left(\frac{1}{\rho_{l}}-\frac{1}{\rho_{g}}\right),
$$

where the flux across the interface $J$ is given by the first-order model used in Preston et al. (2007). 
(ii) The derivative of the potential at infinity must be known. From the Bernoulli equation, we know that

$$
\frac{\partial \phi_{\infty}}{\partial t}+\int_{p_{0}}^{p_{\infty}} \frac{\mathrm{d} p}{\rho}=0
$$

which, applying an analogous procedure as before, gives

$$
\frac{\partial \phi_{\infty}}{\partial t}=-\frac{p_{\infty}-p_{0}}{\rho_{l, 0}},
$$

where $p_{\infty}$ represents the pressure in a region where the potential induced by the bubbles is negligible.

(iii) For a single bubble, $I^{*}$ is zero and the Rayleigh-Plesset equation with the compressibility effects introduced by Keller \& Miksis is recovered.

(iv) The system of equations above simplifies for $c$ tending to infinity to

$$
\begin{gathered}
R_{i} \ddot{R}_{i}+\frac{3}{2} \dot{R}_{i}^{2}=\frac{p_{i}-p_{\infty}}{\rho_{l}}+I^{*}, \\
I^{*}=\sum_{j \neq i}^{N} \frac{\partial \phi_{j}}{\partial t}(0), \\
\frac{\partial \phi_{j}}{\partial t}\left(R_{i}\right)=\frac{R_{j}(t)}{d_{i j}} \frac{\partial \phi_{j}}{\partial t}(t),
\end{gathered}
$$

which recovers the traditional Rayleigh-Plesset equation when the bubble is isolated. Imposing the potential at infinity as zero, it is possible to deduce expressions for the derivative of the potential created by the bubble $j$

$$
\frac{\partial \phi_{j}}{\partial t}\left(R_{i}\right)=\frac{-\ddot{R}_{j} R_{j}^{2}-2 \dot{R}_{j}^{2} R_{j}}{d_{i j}},
$$

which gives the traditional expression for bubble-bubble interactions in incompressible flows

$$
I^{*}=-\sum_{j \neq i}^{N}\left[\frac{\ddot{R}_{j} R_{j}^{2}+2 \dot{R}_{j}^{2} R_{j}}{d_{i j}}\right] .
$$

Also note that to obtain this expression the correction terms coming from the Taylor expansion of the potential evaluated at the interface have been neglected.

\section{REFERENCES}

Akhatov, I., Mettin, R., Ohl, C. D., Parlitz, U. \& Lauterborn, W. 1997 Bjerknes force threshold for stable single bubble sonoluminiscence. Phys. Rev. E 55, 3747-3750.

Akhatov, I., Parlitz, U. \& Lauterborn, W. 1996 Towards a theory of self-organization phenomena in bubble-liquid mixtures. Phys. Rev. E 54 (5), 4990-5003.

Ando, K., Colonius, T. \& Brennen, C. E. 2009 Improvement of acoustic theory of ultrasonic waves in dilute bubbly liquids. J. Acoust. Soc. Am. EL 126, EL69-EL74.

Ando, K., Colonius, T. \& Brennen, C. E. 2011 Numerical simulation of shock propagation in a polydisperse bubbly liquid. Intl J. Multiphase Flow 37, 596-608.

Arora, M., OHL, C. D. \& LOHSE, D. 2007 Effect of nuclei concentration on cavitation cluster dynamics. J. Acoust. Soc. Am. 121 (6), 3432-3436. 
Belytschio, T., Krongauz, Y., Organ, D., Fleming, M. \& Krysl, P. 1996 Meshless methods: an overview and recent developments. Comput. Meth. Appl. Mech. Engng 139 (1-4), 3-47.

Bergmann, R., van der Meer, D., Stijnman, M., Sandtke, M., Prosperetti, A. \& Lohse, D. 2006 Giant bubble pinch-off. Phys. Rev. Lett. 96 (15), 154505-154509.

Best, J. P. \& KuCERA, A. 1992 A numerical investigation of non-spherical rebounding bubbles. J. Fluid Mech. 245 (-1), 137-154.

Blake, J. R., Keen, G. S., Tong, R. P. \& Wilson, M. 1999 Acoustic cavitation: the fluid dynamics of non-spherical bubbles. Phil. Trans. R. Soc. Lond. A 357 (1751), 251-267.

Bremond, N., Arora, M., Dammer, S. M. \& Lohse, D. $2006 a$ Interaction of cavitation bubbles on a wall. Phys. Fluids 18, 121505-121515.

Bremond, N., Arora, M., Ohl, C. D. \& Lohse, D. $2006 b$ Controlled multibubble surface cavitation. Phys. Rev. Lett. 96 (22), 224501-224505.

BREnNen, C. 1995 Cavitation and Bubble Dynamics, p. 254. Oxford University Press, ISBN 0195094093.

Caflisch, R. E., Miksis, M. J., Papanicolaou, G. C. \& Ting, L. 1985 Effective equations for wave propagation in bubbly liquids. J. Fluid Mech. 153, 259-273.

Chahine, G. L. 1993 The final stage of the collapse of a cavitation bubble near a rigid wall. J. Fluid Mech. 257, 147-181.

Chapman, R. B. \& Plesset, M. S. 1970 Thermal Effects in the Free Oscillations of Gas Bubbles. Ft Belvoir Defense Technical information Center.

Climent, E. \& Magnaudet, J. 2006 Dynamics of a two-dimensional upflowing mixing layer seeded with bubbles: bubble dispersion and effect of two-way coupling. Phys. Fluids 18, 103304-103316.

Commander, K. W. \& Prosperetti, A. 1989 Linear pressure waves in bubbly liquids: comparison between theory and experiments. J. Acoust. Soc. Am. 85, 732-746.

Delale, C. F. \& Tryggvason, G. 2008 Shock structure in bubbly liquids: comparison of direct numerical simulations and model equations. Shock Waves 17 (6), 433-440.

DEVIN, C. JR 1959 Survey of thermal, radiation, and viscous damping of pulsating air bubbles in water. Tech. Rep. DTIC Document.

FOLDY, L. L. 1945 The multiple scattering of waves. I. General theory of isotropic scattering by randomly distributed scatterers. Phys. Rev. 67 (3-4), 107-119.

Franck, J. A. \& Colonius, T. 2010 Compressible large-eddy simulation of separation control on a wall-mounted hump. AIAA J. 48, 1098-1107.

Fuster, D., Hauke, G. \& Dopazo, C. 2010 Influence of the accommodation coefficient on nonlinear bubble oscillations. J. Acoust. Soc. Am. 128, 5-10.

Gilmore, F. R. 1952 The Growth or Collapse of a Spherical Bubble in a Viscous Compressible Liquid. http://authors.library.caltech.edu/561/.

Hauke, G., Fuster, D. \& Dopazo, C. 2007 Dynamics of a single cavitating and reacting bubble. Phys. Rev. E 75 (066310), 1-14.

Hinsch, K. 1976 The dynamics of bubble fields in acoustic cavitation. In Proc. 6th Intl Symp. on Nonlinear Acoustics, Moscow 1975, pp. 26-34.

Honein, A. E. \& MoIn, P. 2004 Higher entropy conservation and numerical stability of compressible turbulence simulations. J. Comput. Phys. 201 (2), 531-545.

Ilinskit, Y. A., Hamilton, M. F. \& Zabolotskaya, E. A. 2007 Bubble interaction dynamics in Lagrangian and Hamiltonian mechanics. J. Acoust. Soc. Am. 121, 786-795.

IORDANSKII, S. V. 1960 On the equation of motion for a liquid containing gas bubbles. Zh. Prikl. Mekh. Tekh. Fiz. 3, 102-110.

Johnsen, E. \& COLONiUs, T. 2009 Numerical simulations of non-spherical bubble collapse. J. Fluid Mech. 629, 231-262.

Keller, J. \& Miksis, M. 1980 Bubble oscillations of large amplitude. J. Acoust. Soc. Am. 68 (2), 628-633.

Kogarko, B. S. 1964 One-dimensional unsteady motion of a liquid with an initiation and progression of cavitation. Dokl. Akad. Nauk SSSR 155, 779-782. 
LAUTERBoRn, W. \& BOlLe, H. 1975 Experimental investigations of cavitation-bubble collapse in the neighbourhood of a solid boundary. J. Fluid Mech. 72 (02), 391-399.

LeVeque, R. J. 2002 Finite Volume Methods for Hyperbolic Problems. Cambridge University Press.

Magnaudet, J. \& EAMES, I. 2000 The motion of high-Reynolds-number bubbles in inhomogeneous flows. Annu. Rev. Fluid Mech. 32 (1), 659-708.

Marchioro, M., TANKsley, M. \& Prosperetti, A. 2000 Flow of spatially non-uniform suspensions. Part I: phenomenology. Intl J. Multiphase Flow 26 (5), 783-831.

Mattsson, K. \& NordströM, J. 2004 Summation by parts operators for finite difference approximations of second derivatives. J. Comput. Phys. 199 (2), 503-540.

Mettin, R. \& Lauterborn, W. 2003 Secondary acoustic waves in a polydisperse bubbly medium. J. Appl. Mech. Tech. Phys. 44 (1), 17-26.

Monaghan, J. J. 1982 Why particle methods work. SIAM J. Sci. Stat. Comput. 3, 422.

Oguz, H. N. \& Prosperetti, A. 1990 Bubble entrainment by the impact of drops on liquid surfaces. J. Fluid Mech. 219, 143-179.

Parlitz, U., Mettin, R., Luther, S., Akhatov, I., Voss, M. \& Lauterborn, W. 1999 Spatio-temporal dynamics of acoustic cavitation bubble clouds. Phil. Trans. R. Soc. Lond. A 357 (1751), 313-334.

Peskin, C. S. 2003 The immersed boundary method. Acta Numerica 11, 479-517.

Press, W. H., Teulkolsky, S. A., Vetterling, W. T. \& Flannery, B. P. 1992 Numerical Recipes in Fortran 77. Cambridge University Press.

Preston, A. T., Colonius, T. \& Brennen, C. E. 2007 A reduced order model of diffusive effects on the dynamics of bubbles. Phys. Fluids 19, 1-19. 123302.

Prosperetti, A. 1997 A brief summary of L. van Wijngaarden's work up till his retirement. Appl. Sci. Res. 58 (1), 13-32.

Prosperetti, A., Crum, L. A. \& Commander, K. W. 1988 Nonlinear bubble dynamics. J. Acoust. Soc. Am. 83, 502-514.

Puente, G. F. \& Bonetto, F. J. 2005 Proposed method to estimate the liquid-vapour accommodation coefficient based on experimental sonoluminescence data. Phys. Rev. E 71 (5).

RAYLEIGH, LORD 1917 On the pressure developed in a liquid during the collapse of a spherical cavity. Phil. Mag. 34, 94.

Reisman, G. E, Wang, Y. C. \& Brennen, C. E. 1998 Observations of shock waves in cloud cavitation. J. Fluid Mech. 344, 255.

Schenk, O., Bollhöfer, M. \& RÖMER, R. A. 2008 On large-scale diagonalization techniques for the Anderson model of localization. SIAM Rev. 91-112.

Schenk, O., Waechter, A. \& Hagemann, M. 2007 Matching-based preprocessing algorithms to the solution of saddle-point problems in large-scale nonconvex interior-point optimization. J. Comput. Opt. Appl. 36 (2-3), 321-341.

Seo, J. H., Lele, S. K. \& Tryggvason, G. 2010 Investigation and modelling of bubble-bubble interaction effect in homogeneous bubbly flows. Phys. Fluids 22, 063302.

Storey, B. D. \& Szeri, A. J. 2000 Water vapour, sonoluminiscence and sonochemistry. Proc. R. Soc. Lond. A 456, 1685-1709.

TAnguay, M. 2003 Computation of bubbly cavitating flow in shock wave lithotripsy. PhD thesis, California Institute of Technology, see also URL http://resolver.caltech.edu/CaltechETD: etd-05282004-130028.

Tomar, G., Fuster, D., Zaleski, S. \& Popinet, S. 2010 Multiscale simulations of primary atomization using gerris. Comput. Fluids 39 (4), 1864-1874.

Toro, E. F. 1997 Riemann Solvers and Numerical Methods for Fluid Dynamics - A Practical Introduction. Springer.

Wang, Q. X. \& Blake, R. 2010 Non-spherical bubble dynamics in a compressible liquid. Part 1. Travelling acoustic wave. J. Fluid Mech. 659, 191-224.

WANG, Y. C. \& BRENNEN, C. E. 1999 Numerical computation of shock waves in a spherical cloud of cavitation bubbles. Trans. ASME: J. Fluids Engng 121 (4), 872-880. 
VAN WiJngAARDEN, L. 1964 On the collective collapse of a large number of gas bubbles in water. In Proceedings 11th Int. Cong. Appl. Mech., pp. 854-861.

VAN WIJNGAARDEN, L. 1968 On the equations of motion for mixtures of liquid and gas bubbles. J. Fluid Mech. 33 (3), 465-474.

Xu, N., Apfel, R. E., Khong, A., Hu, X. \& WAng, L. 2003 Water vapour diffusion effects on gas dynamics in a sonoluminescing bubble. Phys. Rev. Lett. E 68 (016309), 1-7.

YASUI, K. 1997 Alternative model of single sonoluminiscence. Phys. Rev. E 56 (6), 6750-6760.

Zhang, D. Z. \& Prosperetti, A. $1994 a$ Averaged equations for inviscid disperse two-phase flow. J. Fluid Mech. 267, 185-219.

Zhang, D. Z. \& Prosperetti, A. $1994 b$ Ensemble phase-averaged equations for bubbly flows. Phys. Fluids 6, 2956-2970.

Zhang, D. Z. \& Prosperetti, A. 1997 Momentum and energy equations for disperse two-phase flows and their closure for dilute suspensions. Intl J. Multiphase Flow 23, 425-453. 